\title{
The impact of vertical human-structure interaction on the response of footbridges to pedestrian excitation
}

\author{
K. Van Nimmen ${ }^{\mathrm{a}, \mathrm{b}}$, G. Lombaert ${ }^{\mathrm{a}}$, G. De Roeck ${ }^{\mathrm{a}}$, P. Van den Broeck ${ }^{\mathrm{a}, \mathrm{b}}$ \\ ${ }^{a}$ KU Leuven, Department of Civil Engineering, Structural Mechanics, B-3001 Leuven, Belgium \\ ${ }^{b}$ KU Leuven, Department of Civil Engineering, Technology Cluster Construction, Structural Mechanics, B-9000 Ghent, Belgium
}

\begin{abstract}
In the present paper, a detailed crowd model is applied to investigate the impact of vertical human-structure interaction (HSI) on the dynamic response of footbridges to pedestrian excitation. Assuming that the walking behaviour of the pedestrian is not affected by the motion of the supporting structure, the contact force between the pedestrian and the supporting structure is decomposed into the force exerted by the pedestrian on a perfectly rigid floor and an interaction term determined by the mechanical interaction between the person and the structure. The model is also used to evaluate the impact of various simplifying assumptions and inter- and intra-person variabilities.

The results show that for the low-frequency dynamic behaviour of footbridges $(<6 \mathrm{~Hz})$, HSI leads to an effective damping ratio which is (significantly) higher than the inherent damping ratio of the empty structure. In addition, it is demonstrated that the detailed moving crowd model can be well approximated by a time-invariant crowd-structure model. Furthermore, the influence of inter- and intra-person variability in step frequency is observed to decrease as the impact of HSI increases. Finally, it is shown that in many cases accounting for HSI leads to a significant reduction in the response of footbridges to pedestrian excitation which may be considered in practical design.
\end{abstract}

Keywords: human-structure interaction, human-induced vibrations, footbridge, random pedestrian traffic, vibration serviceability, full-scale testing

\section{Introduction}

For slender and lightweight structures, vibration serviceability is a matter of growing concern and has often proven critical for design [1]. In recent years, special interest emerged for structures subjected to human-induced loading, in particular for footbridges where the design freedom is restricted by fewer functional and structural demands [2]. Currently, designers are forced to rely on - what are assumed to be 'conservative' - equivalent load models, upscaled from single-person force measurements $[3,4]$. With designs governed by the dynamic performance under high crowd densities, a strong demand exists for the verification and refinement of the currently available load models $[5,6]$.

In the prediction of the structural response induced by groups of pedestrians or crowds, persons crossing the footbridge are often simplified to (moving) loads [7]. This approach disregards the fact that people are mechanical systems and, therefore, does not account for the interaction between the crowd and the structure that is supporting them [8]. However, in the low-frequency range of interest for vibration serviceability $(0-10 \mathrm{~Hz})$, the dynamic behaviour of the human body in vertical direction resembles that of a highly damped single degree of freedom (SDOF) system $[9,10]$ (see figure 1-a) for which the natural frequency $\left(2.7<f_{\mathrm{H} 1}<7.0 \mathrm{~Hz}\right)$ and damping ratio $\left(20<\xi_{\mathrm{H} 1}<60 \%\right)$ largely depend on the body posture $[11,12,13]$. As a result of the interaction between the human body and the footbridge, the dynamic behaviour of the coupled crowd-structure system can differ significantly from that of the empty structure $[14,15,16,17]$. The significance of HSI increases with the

\footnotetext{
${ }^{*}$ Corresponding author. Tel.: +32 (0)9 2658612 .

Email address: katrien.vannimmen@kuleuven.be (K. Van Nimmen)
} 
crowd to structural mass ratio $[18,19,20]$. Hence, the effects of HSI on the structural response to pedestrian excitation are expected to be important for lightweight structures $[8,18]$.

This contribution presents a comprehensive analysis of HSI for pedestrian excitation. To this end, the contact force with the supporting structure is decomposed into the well-known force exerted by a pedestrian on a perfectly rigid laboratory floor $[7,21]$ and a mechanical interaction term which depends on the dynamic properties of both the pedestrian and the footbridge. The pedestrians are thus modelled as passive mechanical systems with an internal driving term that is independent from the motion of the supporting structure. A similar approach is adopted by Alexander [22] and Caprani et al. [23], and is also widely applied in the field of building acoustics to describe source-structure interaction [24]. In the literature, active systems have been developed as well to study the influence of the vibrating surface on the walking behaviour of the crowd [16, 25, 26, 27]. However, since phenomena similar to lock-in have not been observed due to vertical structural vibrations [25], the choice for a passive interaction model has been made in the present study.

The analytical formulation applied in this study is similar to the one introduced by Alexander [22], Caprani et al. [23] and Shahabpoor et al. [28]. However, starting from a general modally reduced system representing the supporting structure, the formulation of the moving crowd model presented here can be readily applied to structures involving both resonant and multi-mode excitation. A comprehensive numerical study involving a realistic crowd model representative of inter-person variability investigates the impact of HSI on the dynamic properties of the coupled crowd-structure system. This numerical analysis is supported by a set of full-scale experimental observations. Furthermore, the impact of HSI on the structural response to pedestrian excitation is analysed for a wide range of pedestrian densities and footbridge parameters, and the impact of modelling simplifications is investigated.

Following the philosophy of the widely-applied design guides Sétra [3] and HiVoSS [4], the structural response to pedestrian excitation is evaluated for the conservative load case where synchronization occurs between one of the natural frequencies of the footbridge and the corresponding harmonic of the walking load. Considering a realistic range of step frequencies $f_{s}[\mathrm{~Hz}]\left(1.0<f_{s}<2.5 \mathrm{~Hz}[1]\right)$, the mean value of the step frequencies (or its 2 nd or 3rd harmonic) of the pedestrians in the crowd is chosen to coincide with the considered natural frequency of the footbridge.

The outline of this paper is as follows. First, the moving crowd model is presented. Second, the effect of HSI on the dynamic properties of the coupled crowd-structure system is investigated and analysed experimentally from in situ measurements on a lightweight steel footbridge. Third, the influence of some common simplifying assumptions and the inter- and intra-person variability in step frequency is investigated. Finally, the structural response to pedestrian excitation is evaluated for a wide range of footbridge parameters and pedestrian densities and the impact of HSI is assessed by comparison with the predictions of a moving force model.

\section{The moving crowd model}

Pedestrians in a crowd are active systems for which the walking behaviour is controlled by an internal driving term. In the crowd-structure model presented here, the pedestrian and the supporting structure (i.e. the footbridge) are considered as two linear subsystems coupled at a single contact point. The contact force not only depends on the internal driving term of the pedestrian but also on the dynamic properties of both subsystems [24]. The equations of motion of the coupled crowd-structure system are derived starting from a general modally reduced system representing the supporting structure. The system presented here can therefore be readily applied to structures involving both resonant and multi-mode excitation or reduced to the case where only a single mode of vibration of the structure is considered. The following subsections subsequently discuss (1) the coupled crowd-structure model, (2) the characteristics of the crowd, (3) the considered statistical approach for the analysis of the predicted structural behaviour and response and (4) a number of modelling simplifications.

\subsection{Coupled crowd-structure model}

A modally reduced system is derived from the linear dynamic finite element (FE) model of the supporting structure. The governing equations of motion in modal coordinates read:

$$
\ddot{\mathbf{z}}(t)+\Gamma \dot{\mathbf{z}}(t)+\boldsymbol{\Omega}^{2} \mathbf{z}(t)=\boldsymbol{\Phi}^{\top} \mathbf{p}(t)
$$


with $\mathbf{z}(t) \in \mathbb{R}^{n_{m}}$ the modal coordinate vector, $n_{m}$ the number of modes retained in the modally reduced system, $\Omega^{2} \in \mathbb{R}^{n_{m} \times n_{m}}$ a diagonal matrix containing the square of the natural frequencies $\omega_{\mathrm{B} j}=2 \pi f_{\mathrm{B} j}$ in $\mathrm{rad} / \mathrm{s}, \Gamma \in \mathbb{R}^{n_{m} \times n_{m}}$ a diagonal matrix containing the terms $2 \xi_{\mathrm{B} j} \omega_{\mathrm{B} j}$ with $\xi_{\mathrm{B} j}[-]$ the modal damping ratios, $\boldsymbol{\Phi} \in \mathbb{R}^{n_{\mathrm{DOF}} \times n_{m}}$ a matrix which has the mass-normalised mode shapes $\boldsymbol{\Phi}_{\mathrm{B} j}$ as columns, $\boldsymbol{\Phi}^{\top} \mathbf{p}(t)$ the modal projection of the external forces $\mathbf{p}(t) \in \mathbb{R}^{n_{\mathrm{DOF}}}$ with $n_{\mathrm{DOF}}$ the number of degrees of freedom of the FE model. The vector of the forces $\mathbf{p}(t)$ is composed of the contact force $(\mathrm{s})$ with the $n_{\mathrm{H}}$ pedestrian $(\mathrm{s})$. For each pedestrian, the contact is reduced to a single point representing at the same time the left and the right foot, an assumption which is justified given the small dimension of the step length relative to the bridge span. The time history of the contact force $p_{\mathrm{p}}(t)$ with each of the $n_{\mathrm{H}}$ pedestrians are collected as rows in the force vector $\mathbf{p}_{\mathbf{p}}(t) \in \mathbb{R}^{n_{\mathrm{H}}}$. The vector of forces applied to the bridge deck $\mathbf{p}(t)$ in Eq. (1) now reads:

$$
\mathbf{p}(t)=\mathbf{S}_{\mathbf{p}}(t) \mathbf{p}_{\mathbf{p}}(t)
$$

where $\mathbf{S}_{\mathbf{p}}(t) \in \mathbb{R}^{n_{\mathrm{DOF}} \times n_{\mathrm{H}}}$ is a selection matrix which transfers the forces to the corresponding DOFs of the FE model of the structure. Each column of the matrix contains a single non-zero element $s_{p}\left(s_{p}=1\right)$ which indicates the time-variant position of the involved pedestrian (see also section 2.2).

At this point, the key assumption is made that the walking behaviour of the pedestrian is not affected by the vibrating surface, i.e. the internal driving term of the pedestrian is identical to the one in case of a stiff supporting system. This assumption holds when the displacements of the bridge deck are (sufficiently) small. In the case where the perceived motion causes the pedestrians to adapt their walking behaviour as for lock-in, this condition is not met. So far, the latter has only been observed for lateral and not for vertical deck motion $[25,29]$. Assuming a fixed driving term allows the body motion and, hence, the contact force of a pedestrian $p_{\mathrm{p}}(t)$, to be decomposed in a term resulting from the driving term $p_{\mathrm{F}}(t)$ and a term resulting from the interaction between the pedestrian and the supporting structure $p_{\mathrm{H}}(t)$ [24] (see also figure 1):

$$
p_{\mathrm{p}}(t)=p_{\mathrm{F}}(t)+p_{\mathrm{H}}(t)
$$

For a perfectly rigid floor, thus without interaction $\left(p_{\mathrm{H}}(t)=0\right)$, the contact force equals the ground reaction forces (GRFs) as registered by the force plates or an instrumented treadmill fixed to a rigid laboratory floor $\left(p_{\mathrm{p}}(t)=p_{\mathrm{F}}(t)\right)[7,30]$. This force term is in this study modelled using the probabilistic single-person force model developed by Živanović et al. [31]. This model accounts for the first five main and intermediate harmonics of the walking load as well as for the narrow-band nature of the force around each (sub-)harmonic. The magnitude of the main and sub harmonics are defined in agreement with the findings of Kerr [32] and Živanović et al. [31], respectively. The related input parameters, i.e. the pedestrian's weight and step frequency, are assigned in agreement with the considered crowd conditions (see subsection 2.2).

When the supporting structure is flexible, the contact force $p_{\mathrm{p}}(t)$ in addition depends on the interaction between the two subsystems [24]. The interaction term $p_{\mathrm{H}}(t)$ in Eq. (3), can be further elaborated based on the mechanical properties of the linear system representing the human body. The latter is represented by a SDOF system (see figure 1-a). The corresponding mechanical properties are discussed in section 2.2. When $p_{\mathrm{H}}(t)$ is written in terms of the displacements of the mass $m_{\mathrm{H} 1}\left(u_{\mathrm{H} 1}(t)\right)$ and the bridge at the contact point $\left(u_{\mathrm{B}}(t)\right)$, Eq. (3) reads:

$$
p_{\mathrm{p}}(t)=p_{\mathrm{F}}(t)-m_{\mathrm{H} 0} \ddot{u}_{\mathrm{B}}(t)+c_{\mathrm{H} 1}\left[\dot{u}_{\mathrm{H} 1}(t)-\dot{u}_{\mathrm{B}}(t)\right]+k_{\mathrm{H} 1}\left[u_{\mathrm{H} 1}(t)-u_{\mathrm{B}}(t)\right]
$$

[Figure 1 about here.]

The second-order differential equations of motion of the moving crowd model can be written as a first-order continuous-time state equation:

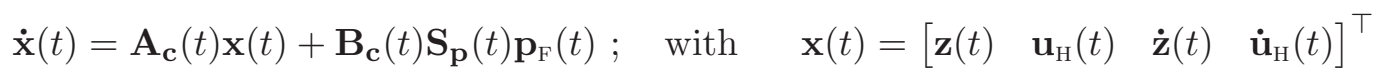

where $\mathbf{x}(t) \in \mathbb{R}^{n_{s}}$ represents the modal state vector and $n_{s}=2\left(n_{m}+n_{\mathrm{H}}\right)$. The time-variant system matrices $\mathbf{A}_{\mathbf{c}}(t) \in \mathbb{R}^{n_{s} \times n_{s}}$ and $\mathbf{B}_{\mathbf{c}}(t) \in \mathbb{R}^{n_{s} \times n_{\text {DOF }}}$ are defined as:

$$
\mathbf{A}_{\mathbf{c}}(t)=\left[\begin{array}{cc}
\mathbf{0} & \mathbf{I} \\
-\overline{\mathbf{M}}_{\mathrm{HB}}^{-1}(t) \overline{\mathbf{K}}_{\mathrm{HB}}(t) & -\overline{\mathbf{M}}_{\mathrm{HB}}^{-1}(t) \overline{\mathbf{C}}_{\mathrm{HB}}(t)
\end{array}\right] ; \quad \mathbf{B}_{\mathbf{c}}(t)=\left[\begin{array}{c}
\mathbf{0} \\
\mathbf{T}_{\mathbf{p}}(t)
\end{array}\right]
$$


where $\mathbf{T}_{\mathbf{p}}(t)$ denotes the generalised input transformation matrix and $\overline{\mathbf{M}}_{\mathrm{HB}}(t), \overline{\mathbf{K}}_{\mathrm{HB}}(t)$ and $\overline{\mathbf{C}}_{\mathrm{HB}}(t)$ are the generalised mass-, stiffness and damping matrices of the coupled crowd-structure system as defined in Appendix A.

The output vector $\mathbf{y}(t) \in \mathbb{R}^{n_{\mathrm{o}}}$ with $n_{\mathrm{o}}$ output quantities is chosen to collect the accelerations of a selection of DOFs of the footbridge $\left(n_{\mathrm{O}} \leq n_{\mathrm{DOF}}\right)$ :

$$
\mathbf{y}(t)=\mathbf{C}_{\mathbf{c}}(t) \mathbf{x}(t)+\mathbf{D}_{\mathbf{c}}(t) \mathbf{S}_{\mathbf{p}}(t) \mathbf{p}_{\mathrm{F}}(t)
$$

with the system matrices $\mathbf{C}_{\mathbf{c}}(t) \in \mathbb{R}^{n_{\mathrm{o}} \times n_{s}}$ and $\mathbf{D}_{\mathbf{c}}(t) \in \mathbb{R}^{n_{\mathrm{o}} \times n_{\mathrm{DOF}}}$ :

$$
\mathbf{C}_{\mathbf{c}}(t)=\mathbf{S}_{\mathrm{o}} \boldsymbol{\Phi}\left[-\overline{\mathbf{M}}_{\mathrm{HB}}^{-1}(t) \overline{\mathbf{K}}_{\mathrm{HB}}(t) \quad-\overline{\mathbf{M}}_{\mathrm{HB}}^{-1}(t) \overline{\mathbf{C}}_{\mathrm{HB}}(t)\right] ; \quad \mathbf{D}_{\mathbf{c}}(t)=\mathbf{S}_{\mathrm{o}} \boldsymbol{\Phi} \mathbf{T}_{\mathbf{p}}(t)
$$

where $\mathbf{S}_{\mathrm{o}} \in \mathbb{R}^{n_{\mathrm{o}} \times n_{\mathrm{DOF}}}$ is a selection matrix that indicates the selected output DOFs of the footbridge.

\subsection{Crowd characteristics}

In a crowd, each pedestrian has his own characteristics, such as the weight, the step frequency and the walking speed (inter-person variability [33]). Some parameters such as the step length and the walking speed may vary along the individual trajectories (intra-person variability $[7,34]$ ). Furthermore, the pedestrians will enter the bridge at different times and their behaviour is subject to various environmental stimuli such as the behaviour of their neighbours and the group as a whole $[35,36]$, as well as the motion of the supporting structure $[29,37]$.

This subsection briefly discusses the characteristics of the crowd and the pedestrian flows as considered next. Given the fact that focus is on the resulting structural response, a number of reasonable simplifying assumptions are made. The comprehensive numerical analysis performed by Tubino et al. [38] shows that the statistical distribution of the walking speed and the pedestrian weight have a negligible influence on the dynamic response of the structure in terms of standard deviation, peak factor and maximum value. Therefore, all pedestrians are assumed to have the same weight $G=700 \mathrm{~N}[3,4,39]$ and walking speed $v_{s}[\mathrm{~m} / \mathrm{s}][3,4]$. The latter is defined in terms of the considered pedestrian density $d$ [pedestrians $\left./ \mathrm{m}^{2}\right][36]$ :

$$
v_{s}(d)=v_{0}\left\{1-\exp \left[-\gamma d_{\mathrm{jam}}\left(\frac{1}{d}-\frac{1}{d_{\mathrm{jam}}}\right)\right]\right\}
$$

where $v=1.34 \mathrm{~m} / \mathrm{s}$ is the free-flow speed, $\gamma=0.354[-]$ a parameter related to the travel purpose and $d_{\text {jam }}=5.4$ pedestrians $/ \mathrm{m}^{2}$ is the jam density, where the corresponding values are set to match the original relation proposed by Weidmann [40]. The walking trajectories $\mathbf{r}(t) \in \mathbb{R}^{1 \times 2}[\mathrm{~m}]$ are assumed to be straight lines parallel to the longitudinal axis of the bridge deck: $\mathbf{r}(t)=\left[r_{1}(t), r_{2}\right]$, whereby the offset in the lateral direction $r_{2}$ is chosen randomly along the bridge deck width and the longitudinal component $r_{1}(t)$ follows from the walking speed and the arrival time of the pedestrian $t_{a}[\mathrm{~s}]: r_{1}(t)=v_{s}\left(t-t_{a}\right)$. The arrival times are assumed to follow a Poisson distribution [35, 41] whereby the arrival rate $\lambda$ [persons/s] is computed from the pedestrian density $d$ [persons $\left./ \mathrm{m}^{2}\right]$ :

$$
\lambda=\frac{n_{\mathrm{H}}}{T}=\frac{A_{\mathrm{eff}} d}{T}
$$

with $n_{\mathrm{H}}[-]$ the number of pedestrians, $T$ [s] denoting the time taken by a pedestrian to cross the bridge span with length $L[\mathrm{~m}]\left(T=L / v_{s}\right)$ and $A_{\text {eff }}\left[\mathrm{m}^{2}\right]$ the walkable bridge deck surface. Together, the pedestrian's step frequency $f_{s}$ and weight $G$ characterise the vertical walking load as exerted on a rigid laboratory floor [7]. To account for intra-person variabilities, the probabilistic single-person force model developed by Živanović et al. [31] is used (see section 2.1).

Aiming at the conservative load case of (near-)resonant human-induced loading, the mean value of the step frequency (or one of its harmonics) is chosen to match one of the natural frequencies of the structure $f_{\mathrm{B} j}$. The fundamental harmonic of the walking load is considered for resonant conditions up to $2.5 \mathrm{~Hz}$, while for natural frequencies between $2.5 \mathrm{~Hz}$ and $5.0 \mathrm{~Hz}$, and between $5.0 \mathrm{~Hz}$ and $7.5 \mathrm{~Hz}$, (near-)resonance occurs with the second and the third harmonic of the walking load, respectively. The present approach accounts for a realistic range of step frequencies $f_{s}[\mathrm{~Hz}]\left(1.0<f_{s}<2.5 \mathrm{~Hz}[1]\right)$ but not for the risk of resonance in relation to the corresponding 
probability distribution of possible step frequencies. Similar to the methodology of the design guides $[3,4]$, the latter could however be accounted for through the application of an associated reduction coefficient on the resulting structural response.

For low pedestrian densities $(d<1)$, spatially unrestricted traffic and thus free movement of the pedestrians is assumed [3]. In the case of dense crowds $(1 \geq d \geq 1.5)$, the normal walking behaviour gets hindered causing the forward movement of the stream to slow down and the level of synchronisation to increase. Beyond the upper limit value of 1.5 persons $/ \mathrm{m}^{2}$, the walking of pedestrians is considered to be obstructed, significantly reducing the dynamic effects [4]. Sparse crowd conditions $(d<1)$ are simulated assuming a Gaussian distribution of the step frequencies, centred around the targeted frequency $f_{l}$ with a standard deviation of $0.175 \mathrm{~Hz}\left(f_{s}=\right.$ $\left.\mathcal{N}\left(f_{l}, 0.175\right)[\mathrm{Hz}][3,4]\right)$ with $1.0<f_{l}<2.5 \mathrm{~Hz}$ and $f_{\mathrm{B}}$ and integer multiple of $f_{l}$. For dense crowd conditions $(1 \geq d \geq 1.5)$, all pedestrians are given the same step frequency $\left(f_{s}=f_{l}[3,4]\right)$. Together, the speed and the pacing rate determine the pedestrian's step length $\left(l_{s}=v_{s} / f_{s}\right)$.

To account for the interaction between the pedestrian and the supporting structure, each pedestrian is in addition represented by a linear mechanical system. To allow for a good representation of the low-frequency $(0-10 \mathrm{~Hz})$ dynamic behaviour of the human body [9, 10, 12, 42], a SDOF model is adopted here (see figure 1a): a sprung mass $m_{\mathrm{H} 1}$, an unsprung mass $m_{\mathrm{H} 0}$ and a spring $k_{\mathrm{H} 1}$ and damping element $c_{\mathrm{H} 1}$ with $m_{\mathrm{H}}=m_{\mathrm{H} 1}+$ $m_{\mathrm{H} 0}=70 \mathrm{~kg}$ and $\mu_{\mathrm{H} 1}=m_{\mathrm{H} 1} / m_{\mathrm{H}}=0.95[11,12]$. The mechanical properties of this SDOF system are set to approximate the low-frequency dynamic behaviour of a person with one or two legs slightly bent $\left(f_{\mathrm{H} 1} \approx 3.25 \mathrm{~Hz}\right.$, $\left.\xi_{\mathrm{H} 1} \approx 0.30[-]\right)$, i.e. similar to the postures assumed during the walking cycle [12, 13]. To account for the expected inter-person variability, the natural frequencies and modal damping ratios for the different individuals are sampled from the following distributions: $f_{\mathrm{H} 1}=\mathcal{N}\left(\mu_{f_{\mathrm{H} 1}}, \sigma_{f_{\mathrm{H} 1}}\right)=\mathcal{N}(3.25,0.32)[\mathrm{Hz}], \xi_{\mathrm{H} 1}=\mathcal{N}\left(\mu_{\xi_{\mathrm{H} 1}}, \sigma_{\xi_{\mathrm{H} 1}}\right)=$ $\mathcal{N}(0.30,0.05)[-][12,13]$. The Gaussian distributions involved in this study are truncated to exclude infeasible negative values. The SDOF system presented here is only an approximation of the human body of a pedestrian and these assumptions should be validated or its model parameters calibrated based on in-field observations. However, the recent study of Shahabpoor [28] involving walking persons reports a similar range of natural frequencies of $2.75-3.00 \mathrm{~Hz}$ and damping ratios of $28-30 \%$.

\subsection{Statistical analysis}

Given the inherent variability of pedestrian excitation (see section 2.2), a statistical analysis seems most suited for the evaluation of the predicted structural behaviour and response [43, 44]. To ensure convergence of the output quantity of interest, a $5 \%$ tolerance is set at $95 \%$ confidence. The number of Monte Carlo samples is thus increased until the probability that the true percentile of the distribution is within $\pm 5 \%$ of the corresponding percentile of the simulated data accumulated so far, is higher than 95\% (Statistics Toolbox, [45]). When the induced structural response is of interest, an observation time period with length $T$ is applied (see section 2.2 and Eq. 10).

\subsection{Modelling simplifications}

The computational cost of solving the time-variant state-space model in Eq. (5) depends on the corresponding system order $n_{s}=2\left(n_{m}+n_{\mathrm{H}}\right)$. Generally, the number of modes $n_{m}$ of the footbridge system is limited and much smaller than the number of pedestrians $n_{\mathrm{H}}$ in case of a (dense) crowd. In the following, a number of simplifying assumptions are discussed (see table 1 and figure 2). The objective is either to reduce the model order or to approximate the dynamic behaviour of the time-variant system by a time-invariant system.

\section{Pseudo moving crowd model}

If the crowd is (roughly) homogeneous and uniformly distributed over the bridge deck, it is reasonable to assume that the dynamic properties of the coupled crowd-structure system are time-invariant. Hence, the coupled crowd-structure system is represented by the time-invariant system matrices $\mathbf{A}_{\mathbf{c}}, \mathbf{B}_{\mathbf{c}}, \mathbf{C}_{\mathbf{c}}$ and $\mathbf{D}_{\mathbf{c}}$ :

$$
\mathbf{A}_{\mathbf{c}}=\left[\begin{array}{cc}
\mathbf{0} & \mathbf{I} \\
-\overline{\mathbf{M}}_{\mathrm{HB}}^{-1} \overline{\mathbf{K}}_{\mathrm{HB}} & -\overline{\mathbf{M}}_{\mathrm{HB}}^{-1} \overline{\mathbf{C}}_{\mathrm{HB}}
\end{array}\right] ; \quad \mathbf{B}_{\mathbf{c}}=\left[\begin{array}{c}
\mathbf{0} \\
\mathbf{T}_{\mathbf{p}}
\end{array}\right] ; \quad \mathbf{C}_{\mathbf{c}}=\mathbf{S}_{\mathrm{o}} \boldsymbol{\Phi}\left[-\overline{\mathbf{M}}_{\mathrm{HB}}^{-1} \overline{\mathbf{K}}_{\mathrm{HB}} \quad-\overline{\mathbf{M}}_{\mathrm{HB}}^{-1} \overline{\mathbf{C}}_{\mathrm{HB}}(t)\right] ; \quad \mathbf{D}_{\mathbf{c}}=\mathbf{S}_{\mathrm{o}} \boldsymbol{\Phi} \mathbf{T}_{\mathbf{p}}
$$

In this way, a pseudo moving crowd model is obtained which accounts for the moving fixed-driver term but approximates the HSI-effects by considering a time-invariant coupled crowd-structure system (see table 1). 


\section{Moving force model}

When HSI-effects are insignificant, the contact force reduces to the fixed-driver term. Consequently, the state vector $\mathbf{x}$ only contains the modal displacements and velocities of the supporting structure and the system matrices $\mathbf{A}_{\mathbf{c}} \in \mathbb{R}^{n_{s} \times n_{s}}, \mathbf{B}_{\mathbf{c}} \in \mathbb{R}^{n_{s} \times n_{m}}, \mathbf{C}_{\mathbf{c}} \in \mathbb{R}^{n_{o} \times n_{s}}$ and $\mathbf{D}_{\mathbf{c}} \in \mathbb{R}^{n_{o} \times n_{\mathrm{DOF}}}$ are independent of the mechanical properties of the pedestrian(s) and, therefore, time-invariant:

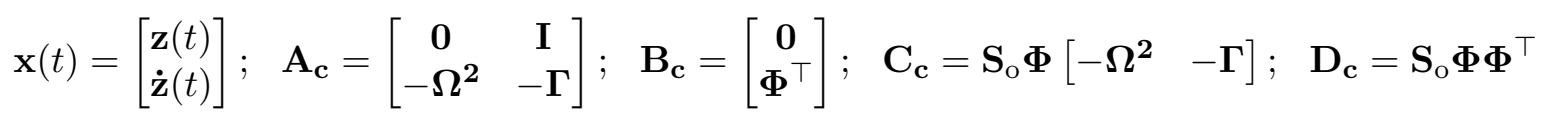

This fixed driver assumption is valid when the natural frequency of the system representing the pedestrian is much more flexible than the supporting structure $\left(f_{\mathrm{H} 1} \lll f_{\mathrm{B} j}\right)$. For these frequency ratios, the subsystems behave decoupled. In this case, one can assume that the pedestrian only injects a force (the corresponding fixeddriver term) into the supporting structure and does not provoke any significant interaction. This assumption is valid for the high-frequency modes of the footbridge $\left(f_{\mathrm{B} j}>10 \mathrm{~Hz}\right)$ which are generally not relevant for the vibration serviceability assessment or when the response of the supporting structure is small. The latter may be the case for non-resonant loading and low pedestrian densities.

\section{Moving force model with added mass}

When the systems representing the pedestrians are much stiffer than the supporting structure $\left(f_{\mathrm{H} 1} \ggg f_{\mathrm{B} j}\right.$ and $u_{\mathrm{H} 1} \approx u_{\mathrm{B}}$ ), the pedestrians act as an equivalent added mass. When the crowd is roughly uniformly distributed on the bridge deck, the response of the coupled crowd-structure system can be computed based on the fixed-driver assumption, i.e. using Eq. (5) with system matrices Eq. (12). The natural frequencies and mode shapes of the supporting structure are in this case computed accounting for the added mass on the bridge deck. This added mass assumption is valid for very low natural frequencies of the footbridge $\left(f_{\mathrm{B} j} \ll 1 \mathrm{~Hz}\right)$. However, such low natural frequencies are generally not found for vertical structural modes.

\section{Stationary force model}

In some cases only the steady-state response is considered whereby it is assumed that for each pedestrian the steady-state response is reached within the time needed to cross the bridge span. However, for the case of (near-)resonant excitation of lowly-damped modes, the latter requires a high number of loading cycles [46] which may not be reached by the actual number of loading cycles $L / l_{s}=L f_{s} / v_{s}$. When in addition HSI is disregarded, the consequent structural response is in this study associated with the stationary force model.

[Table 1 about here.]

[Figure 2 about here.]

\section{The effect of HSI on the dynamic properties of the coupled crowd-structure system}

In some cases, the dynamic behaviour of the coupled crowd-structure system can differ significantly from that of the empty footbridge $[16,18,47]$. As observed for structures equipped with tuned mass dampers [48] and vehicle-bridge interaction [49], the interaction between the two subsystems highly depends on the ratio of the masses and the natural frequencies $[8,50]$. In this section, the position of the crowd is considered fixed. The dynamic properties of the coupled crowd-structure system are examined based on the frequency response function (FRF) relating a harmonic input excitation to the acceleration response of the footbridge, in relation to the corresponding FRF of the empty structure. To take into account inter-person variability, these properties are evaluated by Monte Carlo simulation (see section 2.3). The aim of this analysis is to determine when the interaction is significant and whether or not it is beneficial. At this point, the interaction is considered beneficial if it reduces the maximum resonant steady-state response of the footbridge. The interaction effects evaluated here will be less significant than for other studies based on two-degree-of-freedom systems where inter-person variabilities are disregarded [8].

Section 3.1 discusses the relevant properties of the footbridge and the crowd. Section 3.2 determines the pertinent output quantities, which are subsequently evaluated in section 3.3. The effect of HSI is analysed experimentally in section 3.4 from in situ measurements on a lightweight steel footbridge. 


\subsection{Input parameters}

The following footbridge and crowd parameters are considered:

- Footbridge parameters: A reference structure is used which is considered representative for over 150 recently constructed footbridges [13]. The reference structure has a span of $50 \mathrm{~m}$ and a bridge deck that is $3 \mathrm{~m}$ wide, roughly corresponding to the average values of a bridge deck surface of $150 \mathrm{~m}^{2}$ and a total mass $m_{\text {BRIDGE }}$ of $50 \times 10^{3} \mathrm{~kg}$ [13]. Only the fundamental vertical bending mode is considered with a sinusoidal mode shape and a modal mass equal to half of the total mass of the bridge as for a simply supported beam. As footbridges are generally lowly-damped, modal damping ratios between $0.2 \%$ and $2.0 \%$ are considered. In view of the vibration serviceability issues of footbridges, the natural frequency is varied between $1 \mathrm{~Hz}$ and $6 \mathrm{~Hz}[1,3,4]$.

- Crowd characteristics: Pedestrian densities are usually considered from 15 persons over the entire bridge deck up to 1.5 persons $/ \mathrm{m}^{2}[1,3,4]$. With a total mass of the footbridge $m_{\mathrm{BRIDGE}}=50 \times 10^{3} \mathrm{~kg}$, a bridge deck surface of $150 \mathrm{~m}^{2}$ and an average human body mass of $70 \mathrm{~kg}$ [39], the crowd to bridge mass ratio $\mu_{\mathrm{HB}}[-]$ :

$$
\mu_{\mathrm{HB}}=\frac{m_{\mathrm{CROWD}}}{m_{\mathrm{BRIDGE}}}
$$

reaches a maximum value of about $30 \%$ for a pedestrian density of 1.5 persons $/ \mathrm{m}^{2}$. For the lower $(0.02)$ and upper (0.30) limit of the mass ratios considered here, this corresponds to a total number $n_{\mathrm{H}}$ of 15 and 225 persons, respectively.

As previously discussed in section 2.2, each pedestrian is represented by a SDOF model for a person with one or two legs slightly bent. To account for the inter-person variability, the natural frequencies and modal damping ratios for the different individuals are sampled from the corresponding distributions. In turn, the influence of the location of the pedestrians is taken into account by assigning random pedestrian locations, following a uniform distribution along the bridge deck.

\subsection{Output quantities of interest}

At this point, the dynamic behaviour of the coupled crowd-structure system is of interest. This dynamic behaviour is examined based on the FRF relating a harmonic input excitation to the acceleration response of the footbridge. The FRF of the coupled crowd-structure system $\left(\mathbf{H}_{\mathrm{HB}}\right)$ is found as:

$$
\mathbf{H}_{\mathrm{HB}}(\omega)=\mathbf{D}_{\mathbf{c}}+\mathbf{C}_{\mathbf{c}}\left(\mathrm{i} \omega \mathbf{I}-\mathbf{A}_{\mathbf{c}}\right)^{-1} \mathbf{B}_{\mathbf{c}}
$$

with $\mathbf{A}_{\mathbf{c}}, \mathbf{B}_{\mathbf{c}}, \mathbf{C}_{\mathbf{c}}$ and $\mathbf{D}_{\mathbf{c}}$ the system matrices as defined in equations (6) and (8) which, given the fixed position of the crowd, are in this case time-invariant. Analogously, the FRF of the empty footbridge $\left(\mathbf{H}_{\mathrm{B}}\right)$ can be calculated using equation (14) whereby the system matrices defined in equation 12 can be applied. In case of (near-)resonant excitation, it can be assumed that the structural response is dominated by the contribution of the (near-)resonant mode $j$. As of now, only the (near-)resonant mode of the structure is considered. For this reason, the subscript $j$ is omitted when referring to the corresponding modal properties $\left(f_{\mathrm{B}}, \boldsymbol{\Phi}_{\mathrm{B}}, \xi_{\mathrm{B}}\right.$ and $\left.m_{\mathrm{B}}\right)$ and the FRF of the empty structure can alternatively be written as:

$$
\mathrm{H}_{\mathrm{B}}(\omega)=-\frac{\mathbf{S}_{\mathrm{o}} \boldsymbol{\Phi}_{\mathrm{B}} \boldsymbol{\Phi}_{\mathrm{B}}^{\top}}{\left(-\beta_{\mathrm{B}}^{2}+\mathrm{i} 2 \xi_{\mathrm{B}} \beta_{\mathrm{B}}+1\right)}
$$

with $\beta_{\mathrm{B}}$ the ratio of the harmonic forcing frequency to the natural frequency of the mode $\left(\beta_{\mathrm{B}}=\omega / \omega_{\mathrm{B}}\right)$. From equation (15), it follows that the maximum steady-state amplitude of the response of the empty footbridge $\left(\ddot{u}_{\mathrm{B}, \max }\right)$ is observed for (near-)resonant loading $\left(\omega=\omega_{\mathrm{B}}=2 \pi f_{\mathrm{B}}\right)$ at the antinode of the mode:

$$
\ddot{u}_{\mathrm{B}, \max }=p_{0} \max _{\omega}\left|\mathrm{H}_{\mathrm{B}}(\omega)\right|=p_{0}\left|\mathrm{H}_{\mathrm{B}}\left(\omega_{\mathrm{B}}\right)\right|=\frac{p_{0}}{2 \xi_{\mathrm{B}} m_{\mathrm{B}}}
$$

with $p_{0}[\mathrm{~N}]$ the amplitude of the harmonic input and $\mathbf{S}_{\mathrm{o}} \boldsymbol{\Phi}_{\mathrm{B}} \boldsymbol{\Phi}_{\mathrm{B}}^{\top} \mathbf{S}_{\mathrm{O}}^{\top}=\max \left(\left|\boldsymbol{\Phi}_{\mathrm{B}}\right|\right)^{2}=m_{\mathrm{B}}^{-1}$ with $\mathbf{S}_{\mathrm{o}} \in \mathbb{R}^{n_{\mathrm{DOF}} \times 1}$ in this case indicating the location of the antinode. Apart from the forcing amplitude $p_{0}$, the natural frequency $f_{\mathrm{B}}$, the modal damping ratio $\xi_{\mathrm{B}}$ and the modal mass $m_{\mathrm{B}}$ are the key parameters that determine the structural dynamic response. 
[Figure 3 about here.]

In figure 3, the FRF of the empty footbridge $\left(\mathrm{H}_{\mathrm{B}}\right)$ is compared to that of the coupled crowd-structure system $\left(\mathrm{H}_{\mathrm{HB}}\right)$ for a reference case involving five different frequency ratios $f_{\mathrm{H} 1} / f_{\mathrm{B}}=\{0.4,0.6,0.8,1.0,1.2\}$ and two different mass ratios $\mu_{\mathrm{HB}}=\{0.1,0.4\}$. For the reference case in figure 3 , the modal damping ratio of the footbridge and the modal damping ratio and the natural frequency of the human body models are assigned a fixed value: $\xi_{\mathrm{B}}=0.5 \%, \xi_{\mathrm{H} 1}=30 \%$ and $f_{\mathrm{H} 1}=3.25 \mathrm{~Hz}$, respectively. This figure illustrates that the $\mathrm{FRF} \mathrm{H}_{\mathrm{HB}}(\omega)$ is characterised by a single peak with a value that depends on the frequency ratio $f_{\mathrm{H} 1} / f_{\mathrm{B}}$. The lowest peak value is observed for frequency ratios $f_{\mathrm{H} 1} / f_{\mathrm{B}}$ slightly lower than unity. Furthermore, figure 3 shows that the peak value of the FRF of the coupled crowd-structure system is in all cases significantly lower than the one of the empty footbridge. Comparing figure 3-a with figure 3-b shows that the impact of the interaction, i.e. the change of the peak value and corresponding frequency (abscissa), increases with the mass ratio, as expected. Furthermore, the frequency at which the corresponding peak value of $\mathrm{H}_{\mathrm{Hв}}(\omega)$ is reached, also depends on the frequency ratio $f_{\mathrm{H} 1} / f_{\mathrm{B}}$. For frequency ratios lower and higher than unity, this frequency is found above and below the natural frequency of the empty footbridge, respectively.

Analogous to equation (16), the peak value of the $\mathrm{FRF} \mathrm{H}_{\mathrm{HB}}(\omega)$ is a measure for the maximum amplitude of the steady-state acceleration response of the footbridge $\left(\ddot{u}_{\mathrm{HB}, \max }\right)$ when subjected to harmonic excitation with forcing amplitude $p_{0}$ :

$$
\ddot{u}_{\mathrm{HB}, \max }=p_{0}\left|\mathrm{H}_{\mathrm{HB}}\left(\omega_{\mathrm{HB}}\right)\right|=\frac{p_{0}}{2 \xi_{\text {eff }} m_{\text {eff }}}, \quad \text { with } \quad \omega_{\mathrm{HB}}=\underset{\omega}{\arg \max }\left|\mathrm{H}_{\mathrm{HB}}(\omega)\right|
$$

where $f_{\text {eff }}[\mathrm{Hz}]\left(f_{\text {eff }}=\frac{\omega_{\mathrm{HB}}}{2 \pi}\right), \xi_{\text {eff }}[-]$ and $m_{\text {eff }}[\mathrm{kg}]$ are defined as the effective (or 'apparent') frequency, effective damping and effective mass of the coupled crowd-structure system. The latter is in this study set to match the modal mass of the empty footbridge $\left(m_{\mathrm{eff}}=m_{\mathrm{B}}\right)$, meaning that both changes in mass and damping result into a change in effective damping ratio. By doing so, the effect of HSI is in this section translated only into a change in effective frequency and effective damping ratio.

For each crowd configuration, the normalised effective natural frequency $f_{\text {eff }} / f_{\mathrm{B}}$ and effective damping ratio $\xi_{\text {eff }}$ are calculated.

\subsection{Results}

Figure 4 presents the mean and the $5 \%$ and $95 \%$ percentile value of the normalised effective natural frequency $\left(f_{\text {eff }} / f_{\mathrm{B}}\right)$ and effective damping ratio $\left(\xi_{\text {eff }}\right)$ as a function of the natural frequency of the empty footbridge, for five mass ratios $\mu_{\mathrm{HB}}=\{0.05,0.1,0.2,0.3\}[-]$ and three different modal damping ratios of the empty footbridge $\xi_{\mathrm{B}}=\{0.2,0.5,2.0\} \%$. On average, 500 Monte Carlo simulations are required to attain the desired level of convergence (see section 2.3).

From figure 4, it can be observed that the effective natural frequencies and damping ratios are characterized by a low degree of variation. Exceptions are the modes of the empty footbridge with a natural frequency around $3.5 \mathrm{~Hz}$ for which a large variation in effective natural frequency is observed. The latter is explained by mode shifting whereby the frequency ratio $f_{\text {eff }} / f_{\mathrm{B}}$ determines which mode of the coupled crowd-structure system dominates the structural output $[8,13,51]$.

For low natural frequencies of the empty footbridge $\left(f_{\mathrm{B}}<2.0 \mathrm{~Hz}\right)$, the pedestrians mainly act as an equivalent added mass: the natural frequencies reduce in relation to the considered mass ratio and the effective damping ratio is approximately equal to the modal damping ratio of the empty footbridge $\left(\xi_{\text {eff }} \approx \xi_{\mathrm{B}}\right)$. This is due to the natural frequencies of the human body models that are relatively high in comparison to the natural frequency of the empty footbridge $\left(f_{\mathrm{H} 1} / f_{\mathrm{B}}>1.5\right)$. The effective damping ratio (slightly) increases with the mass ratio and the natural frequency of the empty footbridge. Figure 4 also illustrates that the lower the structural modal mass and the inherent structural damping ratio, the lower the natural frequency from which the increase in effective damping ratio in relation to $\xi_{\mathrm{B}}$ becomes significant.

For intermediate natural frequencies of the empty footbridge $\left(2.0<f_{\mathrm{B}}<4.5 \mathrm{~Hz}\right)$, the frequency ratios are close to unity $\left(f_{\mathrm{H} 1} / f_{\mathrm{B}} \approx 1\right.$ given $\left.\mu_{f_{\mathrm{H} 1}}=3.25 \mathrm{~Hz}\right)$ and the corresponding HSI-effects are expected to be significant. Figures 4 shows that the effective natural frequency of the coupled crowd-structure system is slightly lower (for $f_{\mathrm{B}}<\mu_{f_{\mathrm{H} 1}}$ ) or higher (for $f_{\mathrm{B}}>\mu_{f_{\mathrm{H} 1}}$ ) than the natural frequency of the empty footbridge. More striking is the 
significant increase in the effective damping ratio. For low mass ratios, the effective damping ratio increases up to $4 \%-5 \%$ while for high mass ratios, the effective damping ratio easily exceeds $10 \%$. Similar to the case of a tuned mass damper, the frequency ratio for which the highest effective damping ratio is found, (slightly) decreases for an increasing mass ratio.

For high natural frequencies of the empty footbridge $\left(4.5<f_{\mathrm{B}}<6 \mathrm{~Hz}\right)$, the effective damping ratio again increases with the mass ratio, but less strong than for intermediate natural frequencies. For high mass ratios, the effective natural frequency is also slightly higher than the natural frequency of the empty footbridge while for low mass ratios the HSI-effects are insignificant.

Comparing figures 4-a, 4-b and 4-c shows that the effective damping ratio increases more rapidly for lower values of the inherent structural damping.

[Figure 4 about here.]

\subsection{Experimental observations}

This section examines the effect of HSI based on in situ measurements on a lightweight steel footbridge in Eeklo, Belgium (figure 5). It is verified if the coupled crowd-structure model allows reproducing the experimentally identified effective natural frequency and damping ratio. Tests with standing as well as walking persons have been performed. All procedures were approved by the ethical committee of the university hospital of the KU Leuven and each person gave a written informed consent prior to participation.

For experimental identification of the modal characteristics and the calibration of the numerical model of the Eeklo footbridge, the reader is referred to [52]. The two modes of interest in the present analysis are the fundamental lateral-torsional mode $\left(f_{1}=1.71 \mathrm{~Hz}, \xi_{1}=2.3 \%, m_{1}=34 \times 10^{3} \mathrm{~kg}\right)$ and the first vertical bending mode $\left(f_{2}=2.99 \mathrm{~Hz}, \xi_{2}=0.2 \%, m_{2}=22 \times 10^{3} \mathrm{~kg}\right)$. The results presented in the previous subsection suggest that the second mode to be strongly affected by the presence of persons on the bridge deck.

The structural response is registered during free vibration tests as well as trials involving ambient and random walking excitation. In order to obtain a free vibration dominated by the contribution of a single mode, the bridge is first brought into motion by rhythmical human activities (bobbing) tuned at the selected natural frequency using a metronome. Once the desired level of vibration is reached, the persons cease bobbing and get into the selected body posture, thereby initiating the free vibration phase. The effective natural frequency and damping ratio of the coupled crowd-structure system are subsequently identified by free decay analysis. For the trials involving ambient and random walking excitation, the natural frequency and modal damping ratio of the first and the second mode of the coupled crowd-structure system are identified from an operational modal analysis.

\section{Free decay analysis}

The experiments include trials with a different number of persons on the bridge deck (figure 5). Every individual is positioned at a point where a stringer crosses a transversal beam. In the first setup, only three persons are positioned at midspan. For each of the next setups, the number of persons is increased by six, three on the left and three on the right adjacent cross girder, respectively. For the setups involving free vibration of the fundamental torsional mode, the persons on the central stringer are not included. In addition, the influence of the body posture on the dynamic behaviour of the coupled crowd-structure system is examined. The experiments therefore consider the human body in (1) a normal standing posture and (2) a posture with slightly bent legs. For each of these configurations, four free decays are registered.

[Figure 5 about here.]

[Figure 6 about here.]

In order to estimate the effective damping ratio, an exponential function is fitted to the peak values of the recorded decay with amplitudes between $90 \%$ and $20 \%$ of the maximum peak value of the acceleration levels [53]. Figures 6-b and 6-e illustrate that the applied excitation indeed leads to a free vibration dominated by a single mode, as assumed for the derivation of the effective damping ratio and effective frequency. Figures 6-c and 6-f present the natural logarithm and the least-squares approximation of peak amplitudes $\left(y_{i}\right)$ in terms of 
the number of cycles $\left(x_{i}\right)$. The excellent fit obtained by a linear function with slope $-\delta$, with $\delta$ representing the logarithmic decrement [46], illustrates that the damping characteristics hardly depend on the amplitudes of the recorded free vibration motion.

\section{Operational modal analysis}

For the setups with ambient excitation, 9 or 21 persons were requested to stand still during 3 minutes, all adopting one of the two investigated postures. In addition, the persons were asked to walk at a slow, normal (self-selected) or fast speed along the main span of the bridge during 3 minutes (figure 5). The output-only data have been processed using the reference-based data-driven stochastic subspace identification (SSI-data/ref) algorithm [54]. Previous studies have shown that the estimated modal parameters are subject to a certain degree of uncertainty which is generally low $(\approx 1-2 \%)$ for natural frequencies but can increase up to $50 \%$ for damping ratios [54]. The associated confidence intervals are therefore also presented in the subsequent paragraphs.

\section{Numerical simulations}

The dynamic behaviour of the footbridge is simulated using the mode shapes of the calibrated FE model of the structure and the experimentally identified natural frequencies and modal damping ratios. All individuals involved are weighed in the laboratory to determine their nominal mass. For each trial involving stationary persons, the location of every individual was determined. To account for inter-person variability, the natural frequency $f_{\mathrm{H} 1}$ and damping ratio $\xi_{\mathrm{H} 1}$ of the human body models are sampled from the Gaussian distributions representative for the normal standing posture $\left(f_{\mathrm{H} 1}=\mathcal{N}(5.7,0.56)[\mathrm{Hz}], \xi_{\mathrm{H} 1}=\mathcal{N}(0.44,0.07)[-][10,11]\right)$ and the posture with slightly bent legs (see section 2.2), respectively. From the Monte Carlo simulations, the $5 \%$ and $95 \%$ percentile value of the effective natural frequency and damping ratio of the coupled crowd-structure model are retained.

\section{Results}

Figure 7-a shows the effective frequency and damping ratio of the vertical bending mode for individuals in a normal standing posture, a posture with slightly bent legs and walking at midspan. For the normal standing posture, the frequency ratio is relatively high $\left(f_{\mathrm{H} 1} / f_{\mathrm{B}} \approx[1.5,2]\right)$. As a result, the natural frequency of the footbridge decreases in relation to the added (modal) mass. Despite the low mass ratio, the effective damping increases substantially (from $0.2 \%$ to $0.7 \%$ ) which is owed to the very low inherent damping of the empty structure. The coupled crowd-structure model is able to explain the observed trends. For the posture with slightly bent legs, the frequency ratio is close to unity. As expected, this results in a small modification of the effective frequency and a very large effective damping ratio, e.g. $\xi_{\text {eff }}>3.5 \% \ggg \xi_{\mathrm{B}}=0.2 \%$ when 26 persons are involved. Although small variations of the natural frequencies of the human body models cause a large scatter in the predicted effective damping ratio, the trend is clear and in agreement with the experimentally identified values. The attained effective damping ratio is more than five times higher than for the normal standing posture. These results corroborate the observation of Kasperski [14] where it was found that more damping is induced by a pedestrian than by a passive person for a mode of a footbridge with a natural frequency of $1.8 \mathrm{~Hz}$. Figure 7-a also shows that the effective frequency and damping ratio obtained from the OMA involving walking persons, are in good agreement with the predictions which consider the legs bent posture. This confirms that the presence of moving persons affects the dynamic characteristics of the coupled crowd-structure system in a way which appears to be similar as in the case of stationary persons with two legs bent.

\section{[Figure 7 about here.]}

Figure 7-b shows the effective frequency and damping ratio of the fundamental lateral-torsional mode. For both the normal standing posture and the posture involving two legs slightly bent, the persons act as an additional mass, resulting in a small reduction in effective frequency. The latter can be explained by the fact that, in both cases, the natural frequency of the persons is relatively high compared to the natural frequency of the considered mode $\left(f_{\mathrm{H} 1} / f_{\mathrm{B}}>1.5\right)$. However, figure 7-b shows that, experimentally, an increase of the effective damping ratio is identified as well. It is believed that this mode, which is dominated by lateral motion, is also affected by HSI in the lateral direction. In order to verify this conjecture, a lateral SDOF system for each person with a fundamental natural frequency between $0.5 \mathrm{~Hz}$ and $1.0 \mathrm{~Hz}$ (as suggested by Matsumoto et 
al. [55]) is added to the crowd-structure model. Figure 7-b shows that the considered lateral HSI can explain the observed increase in effective damping.

The results from the OMA involving walking persons are again in good agreement with the predictions which consider the vertical as well as the horizontal body motion.

\section{The effect of HSI on the structural response to pedestrian excitation}

In this final section, focus is on the impact of HSI on the structural response to pedestrian excitation. The maximum structural response is predicted for a wide range of footbridge parameters and pedestrian densities.

First, a number of modelling aspects are discussed. Second, the input parameters of the parametric study are defined and the output quantities of interest are determined. Finally, the interaction effect is examined and the predicted structural response is evaluated.

\subsection{Modelling aspects}

In this first subsection, the influence of common modelling simplifications is investigated. At this point, three different natural frequencies for the fundamental mode of the footbridge $f_{\mathrm{B}}=\{2.00,3.25,5.00\} \mathrm{Hz}$ and two crowd densities $d=\{0.2,1.0\}$ persons $/ \mathrm{m}^{2}$ are considered. The impact of inter-person variability in step frequencies is evaluated through the evaluation of the resulting structural response for two cases involving a different degree of inter-person variability: case $\mathbf{1}$ where the step frequencies are sampled from a Gaussian distribution $\left(f_{s}=\mathcal{N}\left(f_{l}, 0.175\right)\right)$ and case 2 where all pedestrians have the same step frequency $f_{s}=f_{l}$, with $1.0<f_{l}<2.5 \mathrm{~Hz}$ and $f_{\text {eff }}$ an integer multiple of $f_{l}$ (where $f_{\text {eff }}=f_{\mathrm{B}}$ when the empty structure is involved). For the sake of brevity, only (a summary of) the most relevant observations is discussed next.

\section{Impact of modelling simplifications}

The impact of the modelling assumptions involving HSI (see table 1) is evaluated through the comparison of the computational cost, which depends on the system order and whether or not the system is time-variant, and the predicted structural response, i.e. the 95 percentile value of the maximum acceleration level at midspan $\ddot{u}_{\max 95}$ (see table 2 ). The following observations are made:

- The predictions of the SF- and the MF-model correspond well when the number of loading cycles is close to the one required to reach the steady-state response. This number is easily reached when the footbridge is sufficiently long or the walking speed sufficiently low, or, when the required number of load cycles is low and thus when non-resonant loading is involved (case 1) or when the (effective) damping ratio is high.

- The predictions of the MF-model are (slightly) higher than those obtained for the MFAM-model. This difference increases with an increasing pedestrian density.

- The predictions of the PMC- and the MC-model are highly similar, with a difference of at most $5 \%$ for the lowest pedestrian density $\left(0.2\right.$ persons $\left./ \mathrm{m}^{2}\right)$. Indeed, the lower the pedestrian density, the more important the actual spatial distribution of the individuals.

- HSI in most cases significantly reduces the predicted structural response. This reduction is more significant for a higher pedestrian density (and thus mass ratio) and frequency ratios slightly below unity.

- The impact of HSI is most significant for resonant loading (case 2), as also observed for the impact of a tuned mass damper [56].

- Replacing the detailed MC-model by a MF-model reduces the system order, but, this occurs at the expense of a significant loss in accuracy, in particular for high pedestrian densities.

For the reasons stated above, the pseudo moving crowd model is used in the subsequent sections to further assess the impact of HSI.

[Table 2 about here.] 
Inter- and intra-person variability

As (near-)resonant loading is considered, the maximum structural response can be (highly) sensitive to the variabilities in step frequency [44]. The impact of the inter- and intra-person variabilities in step frequency is analysed by comparing the 95 percentile value of the maximum acceleration predicted (A) for case 1 and case 2, and $(\mathrm{B})$ for a driving term $p_{\mathrm{F}}(t)$ modelled using the probabilistic single-person force model (see section 2.1) and a driving term modelled using the continuous load model of Young [57] (disregarding intra-person variabilities). The following observations are made (see also figure 8):

- The impact of intra-person variabilities decreases when the degree of inter-person variability increases: (a high degree of) inter-person variability can compensate for the effect of intra-person variabilities.

- The impact of inter-person and intra-person variabilities decreases for an increasing (effective) damping ratio (as also observed by [58]), thus also for an increasing impact of HSI.

As the impact of intra-person variabilities is not in all cases negligible, in the following the probabilistic singleperson force model developed by Živanović et al. [31] is applied.

[Figure 8 about here.]

\subsection{Input parameters}

In the parametric study, the pseudo moving crowd model is adopted to simulate the structural response in sparse $\left(d<1\right.$ persons $\left./ \mathrm{m}^{2}\right)$ and dense $\left(d \geq 1\right.$ persons $\left./ \mathrm{m}^{2}\right)$ crowd conditions (see section 2.2$)$. The pedestrian flows are generated according to the crowd flow model defined in section 2.2. By comparison with the predictions of the moving force model, it is in addition evaluated in which cases HSI is significant. The following input parameters for the footbridge and the crowd are considered:

- Footbridge parameters: The previously introduced reference structure of a simply supported beam with a span of $50 \mathrm{~m}$ and a bridge deck width of $3 \mathrm{~m}$ is used. Only the contribution of the fundamental mode is considered, with a sinusoidal mode shape, a modal damping ratio $\left(\xi_{\mathrm{B}}\right)$ of $0.2 \%, 0.5 \%$ or $2.0 \%$ and a modal mass $\left(m_{\mathrm{B}}\right)$ ranging from $20 \times 10^{3} \mathrm{~kg}$ up to $50 \times 10^{3} \mathrm{~kg}$. The simulations are performed for a natural frequency $\left(f_{\mathrm{B}}\right)$ in the range between $1.0 \mathrm{~Hz}$ and $6.0 \mathrm{~Hz}$.

- Crowd parameters: Five pedestrian densities are considered: $d=\{0.2,0.5,0.8,1.0,1.5\}$ persons $/ \mathrm{m}^{2}$, corresponding to a total number of 30, 75, 120, 150 and 225 pedestrians, respectively. Following the stochastic load model defined in section 2.2, all pedestrians are given the same step frequency for dense crowd conditions $\left(d \geq 1\right.$ persons $\left./ \mathrm{m}^{2}, f_{s}=f_{l}\right)$, whereas for sparse crowd conditions $\left(d<1\right.$ persons $\left./ \mathrm{m}^{2}\right)$, the step frequencies are sampled from a Gaussian distribution $\left(f_{s}=\mathcal{N}\left(f_{l}, 0.175\right)\right)$. In both cases, the arrival times

follow a Poisson distribution. The impact of HSI is investigated for a realistic crowd model consisting of $n_{\mathrm{H}}$ individuals, with a distribution of human body model parameters representing the inter-person variability (see section 2.2).

\subsection{Output quantities of interest}

For each sample of the Monte Carlo process, the maximum acceleration level over the relevant time window with length $T$ is determined (see section 2.3). In agreement with the widely applied design guides Sétra [3] and HiVoSS $[1,4]$, the 95 percentile value of the maximum predicted acceleration is evaluated here. The factor $R_{\mathrm{HSI}}$ [-] is defined to quantify the reduction of the structural acceleration due to HSI:

$$
R_{\mathrm{HSI}}=\frac{\ddot{u}_{\mathrm{F}, \max 95}}{\ddot{u}_{\mathrm{HSI}, \max 95}}
$$

where $\ddot{u}_{\mathrm{F}, \max 95}$ and $\ddot{u}_{\mathrm{HSI}, \max 95}$ represent the 95 percentile value of the maximum acceleration predicted by the moving force model and the pseudo moving crowd model, respectively. 


\subsection{Results}

As a starting point, the 95 percentile value of the maximum acceleration predicted according to the moving force model $\left(\ddot{u}_{\mathrm{F}, \max 95}\right)$ is discussed for a footbridge with a modal damping ratio of $\xi_{\mathrm{B}}=0.5 \%$, and a low $(0.2$ persons $\left./ \mathrm{m}^{2}\right)$ and a high $\left(1.0\right.$ persons $/ \mathrm{m}^{2}$ ) pedestrian density. The desired degree of convergence (see section 2.3 ) is reached for on average $10^{3}$ up to $10^{4}$ simulations, with an increasing number of required simulations for an increasing synchronization rate and a decreasing effective damping ratio. Figure 9 presents the 95 percentile value of the maximum acceleration levels in terms of the natural frequency and the modal mass of the footbridge. The highest acceleration levels are found for natural frequencies between 1.5 and $2.5 \mathrm{~Hz}$. In this range, resonance occurs with the fundamental harmonic of the walking load. Between $2.5 \mathrm{~Hz}$ and $5.0 \mathrm{~Hz}$, resonance with the second harmonic of the walking load occurs, leading to a lower structural response. Figure 9 also shows that acceleration levels as high as $6 \mathrm{~m} / \mathrm{s}^{2}$ and $20 \mathrm{~m} / \mathrm{s}^{2}$ are predicted for a pedestrian density of 0.2 persons $/ \mathrm{m}^{2}$ and 1.0 persons $/ \mathrm{m}^{2}$, respectively. However, due to (1) the expected increase in structural damping for larger vibration amplitudes and (2) the large structural motion that will hinder the pedestrians in walking, i.e. the self-limiting nature of pedestrian-induced vibrations [25, 59], it is highly unlikely that these extremely high levels will occur in reality. In addition, it is expected that HSI as considered next will significantly reduce the predicted structural response.

\section{[Figure 9 about here.]}

\section{Evaluation of the structural response}

Figure 10 presents the predicted 95 percentile value of the maximum acceleration $\left(\ddot{u}_{\mathrm{HSI}, \max 95}\right)$ in terms of the natural frequency and the modal mass of the footbridge, for the five pedestrian densities and three modal damping ratios of the empty footbridge $\xi_{\mathrm{B}}=\{0.2,0.5,2.0\} \%$. It is again observed that the highest acceleration levels are found for natural frequencies between $1.5 \mathrm{~Hz}$ and $3.0 \mathrm{~Hz}$, i.e. when resonance occurs with the fundamental harmonic of the walking load. A second (smaller) peak is observed for natural frequencies of the footbridge between 4.0 and $5.0 \mathrm{~Hz}$, thus, when the resonance occurs with the second harmonic of the walking load. Similarly to the predictions of the moving force model in figure 9, it is observed from figure 10 that the structural response increases for a decreasing modal damping ratio and a decreasing modal mass of the footbridge. However, the increase observed here is less strong than for the corresponding predictions of the moving force model. This is due to the impact of HSI, leading to a larger effective damping ratio for a low inherent structural damping and a higher mass ratio (see section 3 ).

Figure 10 illustrates that as the modal mass of the footbridge decreases, the natural frequencies at which the highest accelerations are found, slightly increase, in particular for high pedestrian densities. As the modal mass of the footbridge decreases, the ratio of the crowd's to the structural mass increases and, therefore, the effective natural frequency of the coupled crowd-structure system decreases (see section 3). The reduced effective natural frequency may then be situated in the range where resonance occurs with the fundamental harmonic of the walking load.

From figure 10 it can be observed that the maximum peak acceleration levels are no longer necessarily attained for the higher pedestrian densities as it is the case for the moving force model. When taking into account HSI, the governing load case depends on both the mass ratio and the inherent structural damping ratio. For low values of the structural damping ratio, even the lowest pedestrian density may be determinative as this leads to the smallest increase in effective damping ratio. Furthermore, it must be noted that for the considered range of footbridge parameters, the predicted maximum acceleration levels do not exceed $5.0 \mathrm{~m} / \mathrm{s}^{2}$ and, thus, the unrealistically high acceleration levels predicted by the moving force model are not reached, not even in case of high pedestrian densities.

\section{Impact of $H S I$}

Figure 11 presents the reduction factor $R_{\mathrm{HSI}}$ in terms of the natural frequency and the modal mass of the footbridge, for the selected pedestrian densities $\left(d=\{0.2,0.5,0.8,1.0,1.5\}\right.$ persons $\left./ \mathrm{m}^{2}\right)$ and modal damping ratios of the empty footbridge $\left(\xi_{\mathrm{B}}=\{0.2,0.5,2.0\} \%\right)$. It is generally observed that the reduction factor is always equal to or larger than unity, implying that taking into account HSI reduces the predicted structural response. 
Figure 11 clearly illustrates that the reduction factor increases with the pedestrian density, due to the larger impact of HSI (see section 3). For the same reason, the reduction factor is higher for lower modal damping ratios of the empty structure. Moreover, the acceleration levels are most sensitive to the structural damping ratio when purely resonant excitation is considered. Hence, an increase in the effective damping ratio of the coupled crowd-structure system has a larger impact and, thus, larger reduction factor, for dense crowd conditions.

The highest reduction factors in figure 11 are found for natural frequencies of the footbridge between $2.5 \mathrm{~Hz}$ and $5 \mathrm{~Hz}$, which are close to the natural frequency of the human body models. This was expected as the increase in effective damping ratio was found to be most significant for frequency ratios $\left(f_{\mathrm{H} 1} / f_{\mathrm{B}}\right)$ close to unity. The region corresponding to the maximum reduction is slightly shifted towards higher natural frequencies, in particular for high pedestrian densities. This is due to the fact that for the moving force model and natural frequencies between $2.5 \mathrm{~Hz}$ and $3.0 \mathrm{~Hz}$, resonance occurs with the second harmonic of the walking load, whereas for the moving crowd model the effective natural frequency reduces and resonance occurs with the fundamental harmonic of the walking load. Although the amplitude of the fundamental harmonic is significantly larger, the structural response predicted by the moving crowd model is still lower than the one of the moving force model due to HSI. However, the obtained reduction factor is lower than for higher natural frequencies where for both models resonance occurs with the second harmonic of the walking load.

Figure 11 shows that for high pedestrian densities $\left(d \geq 1\right.$ persons $\left./ \mathrm{m}^{2}\right)$, the predicted response has reduced by at least $30 \%$ for the entire range of footbridge parameters. In these cases, HSI is considered significant and may be considered in practical design. Similarly, the reduction is considered significant for sparse pedestrian densities in case of low modal damping ratios $\left(\xi_{\mathrm{B}} \leq 0.5 \%\right)$ and natural frequencies $\left(f_{\mathrm{B}}\right)$ between $2.0 \mathrm{~Hz}$ and $6.0 \mathrm{~Hz}$. On the other hand, for high modal damping ratios of the empty structure $\left(\xi_{\mathrm{B}} \gtrsim 2.0 \%\right)$, the impact of HSI rapidly reduces. Depending on the considered pedestrian density and modal mass, the reduction factor $R_{\mathrm{HSI}}$ stays well below 1.5, particulary for natural frequencies of the empty footbridge $\left(f_{\mathrm{B}}\right)$ below $2.5 \mathrm{~Hz}$ and above $4.5 \mathrm{~Hz}$. In addition, it is noted that a similar comparison with the steady-state acceleration level predicted by a stationary force model would result in even higher values of the reduction factor.

From an engineering point of view, the beneficial effect for taking into account HSI is most desirable when resonance occurs with the fundamental harmonic of the walking load as this leads to the highest structural response. However, the present analysis shows that the effect of HSI is mostly relevant for natural frequencies of the empty footbridge between $2.5 \mathrm{~Hz}$ and $5.0 \mathrm{~Hz}$, when resonance occurs with the second harmonic of the walking load.

The results presented in figure 10 and figure 11 for the fundamental mode of the reference footbridge can be used to assess the sensitivity of the modal contributions to human-induced vibrations and HSI for structures with multiple low-frequency modes.

[Figure 10 about here.]

[Figure 11 about here.]

\section{Conclusions}

A detailed crowd model is applied to investigate the impact of vertical human-structure interaction (HSI) on the dynamic performance of a footbridge exposed to crossing pedestrians. Assuming that the walking behaviour of the pedestrian is not affected by the motion of the supporting structure, the contact force between the pedestrian and the structure is decomposed into (1) the force exerted by the pedestrian on a perfectly rigid floor, and (2) an interaction term which follows from the mechanical interaction between the person and the structure. The pedestrian is represented by a highly damped SDOF system representative of a person with one or two legs slightly bent, corresponding to the body postures assumed during the walking cycle.

The most significant HSI-effect is in the effective damping ratio of the coupled crowd-structure system which is much higher than the inherent damping of the footbridge, in particular for modes with a natural frequency between $2.5 \mathrm{~Hz}$ and $5 \mathrm{~Hz}$ which are close to the natural frequency of the human body. In situ measurements on a lightweight steel footbridge show that the coupled crowd-structure model is able to reproduce the experimentally identified dynamic behaviour. 
The impact of HSI on the response of the footbridge to crossing pedestrians is evaluated for various pedestrian densities and footbridge parameters. By comparison with the results of a moving force model, it is shown that HSI leads to a reduction of the structural response which, in most cases, is significant ( $>30 \%)$. The reduction is most important for structural modes with natural frequencies between $2.5 \mathrm{~Hz}$ and $4.5 \mathrm{~Hz}$ and for low values of the associated modal damping ratio and modal mass. Furthermore, it is demonstrated that simplified force models may lead to unrealistically high acceleration levels. These levels are not reached by the coupled crowd-structure model due to the increase in effective damping ratio.

In addition, the impact of various simplifying assumptions and inter-person variability are evaluated. It is demonstrated that the detailed moving crowd model can be well approximated by a time-invariant crowdstructure model. Furthermore, the influence of the distributions of step frequencies is observed to decrease as the impact of HSI increases.

This study shows that the mechanical interaction with the crowd is relevant for the vertical low-frequency dynamic behaviour of footbridges, leading to a reduction in the structural response which may be considered in practical design. In the assessment of HSI, the (distribution of) mechanical properties of the pedestrians are key parameters. Although the present study is based on reasonable assumptions on the dynamic behaviour of pedestrians and crowds, further research is required for the refinement and verification of these mechanical models.

\section{Acknowledgements}

This research is funded by the Agency for Innovation by Science and Technology in Flanders (IWT) and the Research Foundation Flanders (FWO). The financial support is gratefully acknowledged.

\section{Appendix A: Coupled crowd-structure model}

In section 2, the equations of motion have been written for the human body model and the supporting structure separately. They are combined by eliminating the interaction forces $\mathbf{p}_{\mathrm{H}}(t)$. The displacements, velocities and accelerations of the bridge at the contact point with a pedestrian $k$ are found as:

$$
u_{\mathrm{B}}(t)=\mathbf{S}_{\mathbf{p} k}^{\top}(t) \Phi \mathbf{z}(t), \quad \dot{u}_{\mathrm{B}}(t)=\mathbf{S}_{\mathbf{p} k}^{\top}(t) \boldsymbol{\Phi} \dot{\mathbf{z}}(t), \quad \ddot{u}_{\mathrm{B}}(t)=\mathbf{S}_{\mathbf{p} k}^{\top}(t) \Phi \ddot{\mathbf{z}}(t)
$$

with $\mathbf{S}_{\mathbf{p} k}(t)$ the corresponding column of the matrix $\mathbf{S}_{\mathbf{p}}(t)$. The interaction force $p_{\mathrm{H}}(t)$ can be written as:

$$
p_{\mathrm{H}}(t)=k_{\mathrm{H} 1}\left[u_{\mathrm{H} \mathbf{1}}(t)-\mathbf{S}_{\mathbf{p} k}^{\top}(t) \mathbf{\Phi} \mathbf{z}(t)\right]+c_{\mathrm{H} 1}\left[\dot{u}_{\mathrm{H} \mathbf{1}}(t)-\mathbf{S}_{\mathbf{p} k}^{\top}(t) \boldsymbol{\Phi} \dot{\mathbf{z}}(t)\right]-m_{\mathrm{H} 0} \mathbf{S}_{\mathbf{p} k}^{\top}(t) \boldsymbol{\Phi} \ddot{\mathbf{Z}}(t)
$$

Subsequently, Eq. (A.2) and the equations of motion for each of the $n_{\mathrm{H}}$ human body models:

$$
m_{\mathrm{H} 1} \ddot{u}_{\mathrm{H} 1}(t)+c_{\mathrm{H} 1}\left[\dot{u}_{\mathrm{H} 1}(t)-\dot{u}_{\mathrm{B}}(t)\right]+k_{\mathrm{H} 1}\left[u_{\mathrm{H} 1}(t)-u_{\mathrm{B}}(t)\right]=0
$$

are introduced in the equations of motion of the supporting structure Eq. (1):

$$
\overline{\mathbf{M}}_{\mathrm{B}}(t) \ddot{\mathbf{z}}(t)+\overline{\mathbf{C}}_{\mathrm{B}}(t) \dot{\mathbf{z}}(t)+\overline{\mathbf{K}}_{\mathrm{B}}(t) \mathbf{z}(t)=\boldsymbol{\Phi}^{\top} \mathbf{S}_{\mathbf{p}}(t) \mathbf{p}_{\mathrm{F}}(t)+\boldsymbol{\Phi}^{\top} \mathbf{S}_{\mathbf{p}}(t)\left[\mathbf{K}_{\mathrm{H}} \mathbf{u}_{\mathrm{H} 1}(t)+\mathbf{C}_{\mathrm{H}} \dot{\mathbf{u}}_{\mathrm{H} 1}(t)\right]
$$

where the generalised mass $\overline{\mathbf{M}}_{\mathrm{B}}(t) \in \mathbb{R}^{n_{m} \times n_{m}}$, damping $\overline{\mathbf{C}}_{\mathrm{B}}(t) \in \mathbb{R}^{n_{m} \times n_{m}}$ and stiffness $\overline{\mathbf{K}}_{\mathrm{B}}(t) \in \mathbb{R}^{n_{m} \times n_{m}}$ matrices read:

$$
\overline{\mathbf{M}}_{\mathrm{B}}(t)=\mathbf{I}+\boldsymbol{\Phi}^{\top} \mathbf{S}_{\mathbf{p}}(t) \mathbf{M}_{\mathrm{H} 0} \mathbf{S}_{\mathbf{p}}^{\top}(t) \boldsymbol{\Phi} ; \quad \overline{\mathbf{C}}_{\mathrm{B}}(t)=\boldsymbol{\Gamma}+\boldsymbol{\Phi}^{\top} \mathbf{S}_{\mathbf{p}}(t) \mathbf{C}_{\mathrm{H}} \mathbf{S}_{\mathbf{p}}^{\top}(t) \boldsymbol{\Phi} ; \quad \overline{\mathbf{K}}_{\mathrm{B}}(t)=\boldsymbol{\Omega}^{2}+\boldsymbol{\Phi}^{\top} \mathbf{S}_{\mathbf{p}}(t) \mathbf{K}_{\mathrm{H}} \mathbf{S}_{\mathbf{p}}^{\top}(t) \boldsymbol{\Phi}
$$

with:

$$
\mathbf{M}_{\mathrm{H} 0}=\operatorname{diag}\left\{\mathbf{m}_{\mathrm{H} \mathbf{0}}\right\} ; \quad \mathbf{C}_{\mathrm{H}}=\operatorname{diag}\left\{\mathbf{c}_{\mathrm{H} \mathbf{1}}\right\} ; \quad \mathbf{K}_{\mathrm{H}}=\operatorname{diag}\left\{\mathbf{k}_{\mathrm{H} \mathbf{1}}\right\}
$$

The terms in $\mathbf{u}_{\mathrm{H} 1}(t)$ and $\dot{\mathbf{u}}_{\mathrm{H} 1}(t)$ are moved to the left hand side of Eq. (A.4). By combining the two sets of equations of motion, Eq. (A.4) and Eq. (A.3), in a single system, the coupled crowd-structure system is written in state-space form. The continuous-time state-space model is given by the following system of equations:

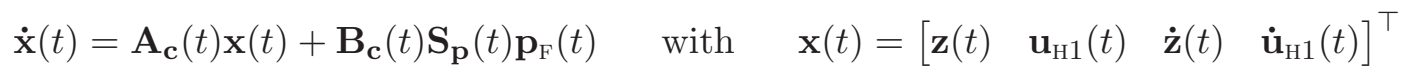


where the state vector $\mathbf{x}(t) \in \mathbb{R}^{n_{s}}$ with $n_{s}=2\left(n_{m}+n_{\mathrm{H}}\right)$ collects the modal displacements and velocities of the footbridge $(\mathbf{z})$, and the displacements and velocities of the human body models $\left(\mathbf{u}_{\mathrm{H} 1}\right)$. The system matrices $\mathbf{A}_{\mathbf{c}}(t) \in \mathbb{R}^{n_{s} \times n_{s}}$ and $\mathbf{B}_{\mathbf{c}}(t) \in \mathbb{R}^{n_{s} \times n_{\text {DOF }}}$ are defined as:

$$
\mathbf{A}_{\mathbf{c}}(t)=\left[\begin{array}{cc}
\mathbf{0} & \mathbf{I} \\
-\overline{\mathbf{M}}_{\mathrm{HB}}^{-1}(t) \overline{\mathbf{K}}_{\mathrm{HB}}(t) & -\overline{\mathbf{M}}_{\mathrm{HB}}^{-1}(t) \overline{\mathbf{C}}_{\mathrm{HB}}(t)
\end{array}\right] ; \quad \mathbf{B}_{\mathbf{c}}(t)=\left[\begin{array}{c}
\mathbf{0} \\
\mathbf{T}_{\mathbf{p}}(t)
\end{array}\right] \quad \text { with } \quad \mathbf{T}_{\mathbf{p}}(t)=\left[\begin{array}{c}
\overline{\mathbf{M}}_{\mathrm{B}}^{-\mathbf{1}}(t) \boldsymbol{\Phi}^{\top} \\
\mathbf{0}
\end{array}\right]
$$

where the matrices $\overline{\mathbf{M}}_{\mathrm{HB}}(t), \overline{\mathbf{K}}_{\mathrm{HB}}(t)$ and $\overline{\mathbf{C}}_{\mathrm{HB}}(t) \in \mathbb{R}^{n_{\mathrm{HB}} \times n_{\mathrm{HB}}}$ with $n_{\mathrm{HB}}=n_{m}+n_{\mathrm{H}}$, refer to the generalised mass-, stiffness and damping matrices of the coupled crowd-structure system:

$$
\begin{aligned}
& \overline{\mathbf{M}}_{\mathrm{HB}}(t)=\left[\begin{array}{cc}
\overline{\mathbf{M}}_{\mathrm{B}}(t) & \mathbf{0} \\
\mathbf{0} & \mathbf{M}_{\mathrm{H}}
\end{array}\right] \quad \text { with } \quad \mathbf{M}_{\mathrm{H}}=\operatorname{diag}\left\{\mathbf{m}_{\mathrm{H} \mathbf{1}}\right\} ; \\
& \overline{\mathbf{C}}_{\mathrm{HB}}(t)=\left[\begin{array}{cc}
\overline{\mathbf{C}}_{\mathrm{B}}(t) & -\boldsymbol{\Phi}^{\top} \mathbf{S}_{\mathbf{p}}(t) \mathbf{C}_{\mathrm{H}} \\
-\mathbf{C}_{\mathrm{H}} \mathbf{S}_{\mathbf{p}}^{\top}(t) \boldsymbol{\Phi} & \mathbf{C}_{\mathrm{H}}
\end{array}\right] ; \quad \overline{\mathbf{K}}_{\mathrm{HB}}(t)=\left[\begin{array}{cc}
\overline{\mathbf{K}}_{\mathrm{B}}(t) & -\boldsymbol{\Phi}^{\top} \mathbf{S}_{\mathbf{p}}(t) \mathbf{K}_{\mathrm{H}} \\
-\mathbf{K}_{\mathrm{H}} \mathbf{S}_{\mathbf{p}}^{\top}(t) \boldsymbol{\Phi} & \mathbf{K}_{\mathrm{H}}
\end{array}\right]
\end{aligned}
$$

\section{References}

[1] C. Butz, M. Feldmann, C. Heinemeyer, and G. Sedlacek, "SYNPEX: Advanced load models for synchronous pedestrian excitation and optimised design guidelines for steel footbridges," Technical Report, Research Fund for Coal and Steel, 2008.

[2] A. Keil, "Footbridge design - reflecting on objectives," in Proceedings of the 5th International Footbridge Conference (L. Debell and H. Russel, eds.), (London, UK), pp. 22-33, July 2014.

[3] Association Française de Génie Civil, Sétra/AFGC, Sétra: Evaluation du comportement vibratoire des passerelles piétonnes sous l'action des piétons (Assessment of vibrational behaviour of footbridges under pedestrian loading), 2006.

[4] C. Heinemeyer, C. Butz, A. Keil, M. Schlaich, A. Goldack, M. Lukić, B. Chabrolin, A. Lemaire, P. Martin, A. Cunha, and E. Caetano, Design of Lightweight Footbridges for Human Induced Vibrations - Background document in support to the implementation, harmonization and further development of the Eurocodes. JRC-ECCS 2009, 2009.

[5] C. T. Georgakis and E. Ingólfsson, "Recent advances in our understanding of vertical and lateral footbridge vibrations," in Proceedings of the 5th International Footbridge Conference, (London, UK), July 2014.

[6] S. Živanović, A. Pavić, and E. Ingólfsson, "Modelling spatially unrestricted pedestrian traffic on footbridges," Journal of Structural Engineering, vol. 136, no. 10, pp. 1296-1308, 2010.

[7] V. Racić, A. Pavić, and P. Reynolds, "Experimental identification and analytical modelling of walking forces: a literature review," Journal of Sound and Vibration, vol. 326, pp. 1-49, 2009.

[8] R. Sachse, A. Pavić, and P. Reynolds, "Parametric study of modal properties of damped two-degree-of-freedom crowd-structure dynamic systems," Journal of Sound and Vibration, vol. 274, pp. 461-480, 2004.

[9] International Organisation for Standardization, ISO 5982:1981 Vibration and shock - Mechanical driving point impedance of the human body, 1981.

[10] J. M. W. Brownjohn, "Energy dissipation from vibration floor slabs due to human-structure interaction," Journal of Shock and Vibration, vol. 8, pp. 315-323, 2001.

[11] Y. Matsumoto and M. J. Griffin, "Dynamic response of the standing human body exposed to vertical vibration: influence of posture and vibration magnitude," Journal of Sound and Vibration, vol. 212, no. 1, pp. 85-107, 1998.

[12] Y. Matsumoto and M. J. Griffin, "Mathematical models for the apparent masses of standing subjects exposed to vertical whole-body vibration," Journal of Sound and Vibration, vol. 260, pp. 431-451, 2003.

[13] K. Van Nimmen, G. Lombaert, G. De Roeck, and P. Van den Broeck, "Human-induced vibrations of footbridges: The effect of vertical human-structure interaction," in Proceedings of IMAC 34, the International Modal Analysis Conference, (Orlando, Florida, US), Springer-Verlag, January - February 2016.

[14] M. Kasperski, "Damping induced by pedestrians," in Proceedings of the 8th International Conference on Structural Dynamics of EURODYN, (Porto, Portugal), June-July 2014.

[15] G. Busca, A. Cappellini, S. Manzoni, M. Tarabini, and M. Vanali, "Quantification of chances in modal parameters due to the presence of passive people on a slender structure," Journal of Sound and Vibration, vol. 333, pp. 5641-5652, 2014.

[16] M. Bocian, J. H. G. Macdonald, and J. F. Burn, "Biomechanically-Inspired Modelling of Pedestrian-Induced Vertical SelfExcited Forces," Journal of Bridge Engineering, vol. 18, pp. 1336-1346, 2013.

[17] L. Pedersen, "Implications of interaction between humans and structures," in Proceedings of IMAC 33, the International Modal Analysis Conference, (Orlando, Florida, US), Springer-Verlag, January - February 2015.

[18] E. Agu and M. Kasperski, "Influence of the random dynamic parameters of the human body on the dynamic characteristics of the coupled system structure-crowd," Journal of Sound and Vibration, vol. 330, no. 3, pp. 431-444, 2011.

[19] C. A. Jones, P. Reynolds, and A. Pavić, "Vibration serviceability of stadia structures subjected to dynamic crowd loads: a literature review," Journal of Sound and Vibration, vol. 330, pp. 1531-1566, 2011.

[20] J. W. Dougil, J. R. Wright, J. G. Parkhouse, and R. E. Harrison, "Human structure interaction during rhytmic bobbing," The Structural Engineer, pp. 32-39, 2006.

[21] A. Ebrahimpour, A. Hamam, R. Sack, and W. Patten, "Measuring and Modeling Dynamic Loads imposed by Moving Crowds," Journal of Structural Engineering, no. 122, pp. 1468-1474, 1996. 
[22] N. Alexander, "Theoretical treatment of crowd-structure interaction dynamics," Proceedings of the Institution of Civil Engineers: Structures and Buildings, vol. 159, no. 6, pp. 329-338, 2006.

[23] C. Caprani, J. Keogh, P. Archbold, and P. Fanning, "Characteristic vertical response of a footbridge due to crowd loading," in Proceedings of the 8th International Conference on Structural Dynamics of EURODYN, (Leuven, Belgium), July 2011.

[24] F. Fahy and P. Gardonio, Sound and structural vibration: Radiation, transmission and response. Academic press, Second edition, 2007.

[25] E. T. Ingólfsson, C. T. Georgakis, and J. Jönsson, "Pedestrian-induced lateral vibrations of footbridges: A literature review," Engineering Structures, vol. 45, pp. 21-52, 2012.

[26] S. Erlicher, A. Trovato, and P. Argoul, "Modelling the lateral pedestrian force on a rigid floor by a self-sustained oscillator," Mechanical Systems and Signal Processing, vol. 24, pp. 1579-1604, 2010.

[27] J. W. Qin, S. S. Law, Q. S. Yang, and N. Yang, "Pedestrian-bridge dynamic interaction, including human participation," Journal of Sound and Vibration, vol. 332, pp. 1107-1124, 2013.

[28] E. Shahabpoor, A. Pavić, and V. Racić, "Identification of mass-spring-damper model of walking humans," Structures, vol. 5, pp. 233-246, 2016.

[29] M. Bocian, J. H. G. Macdonald, and J. F. Burn, "Biomechanically inspired modelling of pedestrian-induced forces on laterally oscillating structures," Journal of Sound and Vibration, vol. 331, pp. 3914-3929, 2012.

[30] S. C. Kerr and N. W. M. Bishop, "Human induced loading on flexible staircases," Engineering Structures, vol. 23, no. 1, pp. 37-45, 2001.

[31] S. Živanović, A. Pavić, and P. Reynolds, "Probability-based prediction of multi-mode vibration response to walking excitation," Engineering Structures, vol. 29, no. 6, pp. 942-954, 2007.

[32] S. C. Kerr, Human induced loading of staircases. PhD thesis, University College London, Mechanical Engineering Department, 1998.

[33] C. C. Caprani, J. Keogh, P. Archbold, and P. Fanning, "Enhancement for the vertical response of footbridges subjected to stochastic crowd loading," Computers and Structures, vol. 102-103, pp. 87-96, 2012.

[34] C. Sahnaci and M. Kasperski, "Simulation of random pedestrian flow," in Proceedings of the 8th International Conference on Structural Dynamics of EURODYN, (Leuven, Belgium), July 2011.

[35] D. Helbing and P. Molnar, "Social force model for pedestrian dynamics," Physical Review, vol. 51, no. 5, pp. 4282-4286, 1995.

[36] L. Bruno and F. Venuti, "Crowd-structure interaction in footbridges: Modelling, application to real case-study and sensitivity analysis," Journal of Sound and Vibration, vol. 323, pp. 475-493, 2009.

[37] S. P. Carroll, J. S. Owen, and M. F. M. Hussein, "Modelling crowd-bridge dynamic interaction with a discrete defined crowd," Journal of Sound and Vibration, vol. 331, no. 11, pp. 2685-2709, 2012.

[38] F. Tubino and G. Piccardo, "Serviceability assessment of footbridges in unrestricted pedestrian traffic conditions," Structure and Infrastructure Engineering, 2016.

[39] S. C. Walpole, D. Prieto-Merino, P. Edwards, J. Cleland, G. Stevens, and I. Roberts, "The weight of nations: an estimation of adult human biomass," BMC Public Health, vol. 12, p. 439, 2012.

[40] U. Weidmann, "Transporttechnik der fussganger (Transport technology of the pedestrian)," Schriftenreihe IVT-Berichte, vol. 90, 1993.

[41] S. Živanović, "Benchmark footbridge for vibration serviceability assessment under vertical component of pedestrian load," Journal of Structural Engineering, vol. 138, pp. 1193-1202, 2012.

[42] X. Zheng and J. M. W. Brownjohn, "Modelling and simulation of human-floor system under vertical vibration," in Proceedings of SPIE: Smart Structures and Material (L. Davis, ed.), 4327, pp. 513-520, 2001.

[43] Q. Li, J. Fan, J. Nie, Q. Li, and Y. Chen, "Crowd-induced random vibration of footbridge and vibration control using multiple tuned mass dampers," Journal of Sound and Vibration, no. 329, pp. 4068-4092, 2010.

[44] G. Piccardo and F. Tubino, "Equivalent spectral model and maximum dynamic response for the serviceability analysis of footbridges," Engineering Structures, vol. 40, pp. 445-456, 2012.

[45] MATLAB, Statistics Toolbox (R2014a). Natick, Massachusetts: The MathWorks Inc., 2014.

[46] A. K. Chopra, Dynamics of structures: Theory and applications to earthquake engineering. Prentice Hall, 1995.

[47] W. Dong, M. Kasperski, and G. Shiqiao, "Change of the dynamic characteristics of a pedestrian bridge during a mass event," in Proceedings of the 8th International Conference on Structural Dynamics of EURODYN, (Leuven, Belgium), July 2011.

[48] F. Weber, G. Feltrin, and H. Olaf, Samco Final Report - F05 Guidelines for Structural Control. Structural engineering research laboratory, Swiss federal laboratories for materials testing and research, 2006.

[49] A. Doménech, P. Museros, and M. Martínez-Rodrigo, "Influence of the vehicle model on the prediction of the maximum bending response of simply supported bridges under high-speed railway traffic," Engineering Structures, vol. 72, pp. 123-139, 2014.

[50] J. P. Den Hartog, Mechanical Vibrations. Dover, 1985.

[51] D. J. Ewins, Modal testing: Theory, Practice and Application. Research Studies Press, Baldock, UK, 2000.

[52] K. Van Nimmen, G. Lombaert, I. Jonkers, G. De Roeck, and P. Van den Broeck, "Characterisation of walking loads by 3D inertial motion tracking," vol. 333, pp. 5212-5226, 2014.

[53] F. Magalhães, A. Cunha, E. Caetano, and R. Brincker, "Damping estimation using free decays and ambient vibration tests," Mechanical Systems and Signal Processing, vol. 24, pp. 1274-1290, 2010.

[54] E. Reynders, R. Pintelon, and G. De Roeck, "Uncertainty bounds on modal parameters obtained from Stochastic Subspace Identification," Mechanical Systems and Signal Processing, vol. 22, no. 4, pp. 948-969, 2008.

[55] Y. Matsumoto and M. J. Griffin, "The horizontal apparent mass of the standing human body," Journal of Sound and Vibration, vol. 330, pp. 3284-3297, 2011. 
[56] K. Van Nimmen, P. Verbeke, G. Lombaert, G. De Roeck, and P. Van den Broeck, "Numerical and experimental evaluation of the dynamic performance of a footbridge with tuned mass dampers," Journal of Bridge Engineering, 2016.

[57] M. R. Willford, C. Field, and P. Young, "Improved methodologies for the prediction of footfall-induced vibration," ASCE Building Integration Solutions, pp. 1-15, 2004.

[58] S. Krenk, "Dynamic response to pedestrian loads with statistical frequency distribution," Journal of Egineering Mechanics, vol. 138, pp. 1275-1281, 2012.

[59] S. Nakamura, "Model for lateral excitation of footbridges by synchronous walking," ASCE Journal of Structural Engineering, vol. 130, no. 1, pp. 32-37, 2011. 


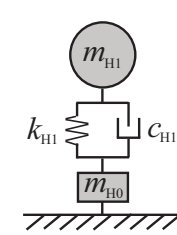

(a)

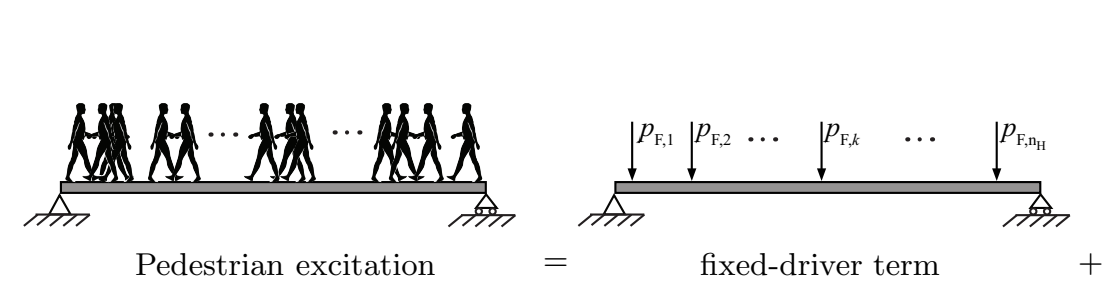

(b)

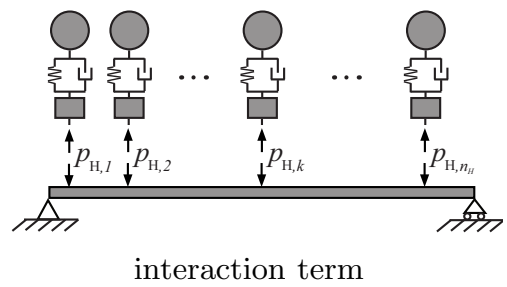

(d)

Figure 1: Schematic representation of (a) the human body model, (b) a footbridge subjected to pedestrian excitation and the proposed decomposition of the contact force $\mathbf{p}_{\mathrm{P}}$ into (c) the fixed-driver term $\mathbf{p}_{\mathrm{F}}$ and $(\mathrm{d})$ the interaction term $\mathbf{p}_{\mathrm{H}}$. 


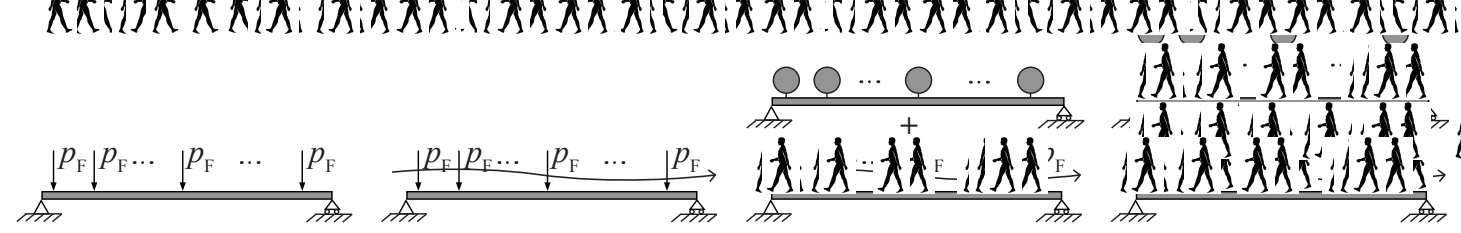

(a) $\mathrm{SF}$

(b) MF

(c) MFAM

(d) PMC

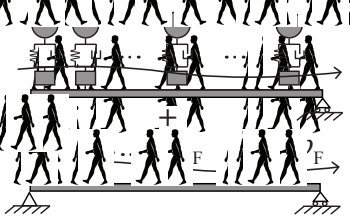

(e) MC

Figure 2: Schematic representation of the models considered in this paper to predict the structural response to pedestrian excitation: (a) Stationary force model (SF), (b) Moving force model (MF), (c) Moving force model with added mass (MFAM), (d) Pseudo moving crowd model (PMC) and (e) Moving crowd model (MC). 


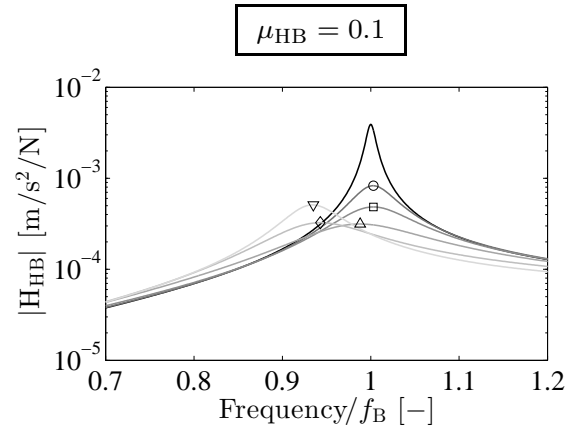

(a)

Figure 3: The FRF $\mathrm{H}_{\mathrm{HB}}(\omega)$ of the coupled crowd-structure system with $\xi_{\mathrm{B}}=0.5 \%, \xi_{\mathrm{H} 1}=30 \%$ and a mass ratio of (a) $\mu_{\mathrm{HB}}=0.1$ and (b) $\mu_{\mathrm{HB}}=0.4$, for a frequency ratio $f_{\mathrm{H} 1} / f_{\mathrm{B}}$ of: $0.4(\circ), 0.6(\square), 0.8(\Delta), 1.0(\diamond)$ and $1.2(\nabla)$ and of the empty structure (black). 

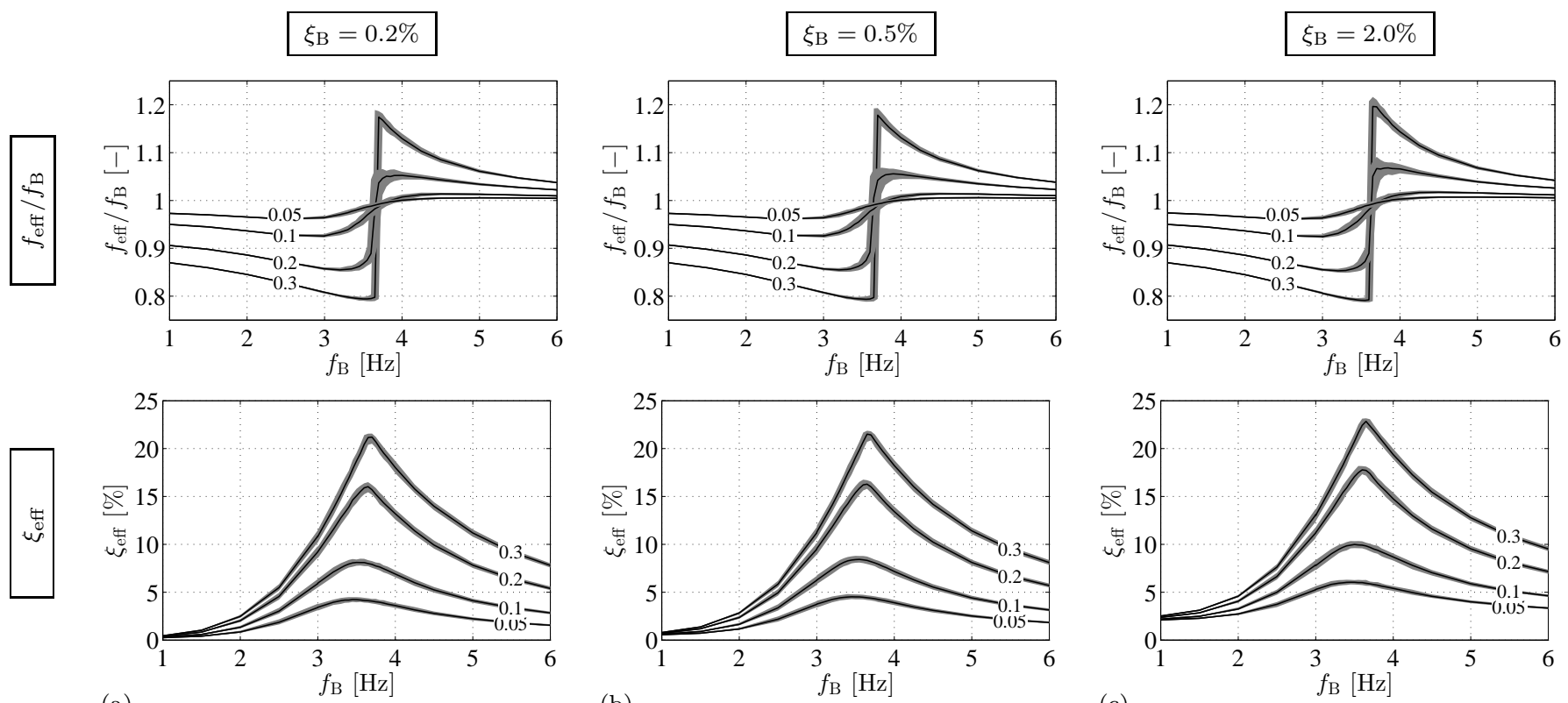

(b)

(c)

Figure 4: The mean value (black) and the 5\%-95\% percentile interval (grey) of the normalised effective frequency $f_{\text {eff }} / f_{\mathrm{B}}($ top) and the effective damping ratio $\xi_{\text {eff }}$ (bottom) of the coupled crowd-structure system in terms of the natural frequency of the footbridge $\left(f_{\mathrm{B}}\right)$, for the mass ratios $\mu_{\mathrm{HB}}=\{0.05,0.1,0.2,0.3\}$ and a footbridge with a modal damping ratio $\xi_{\mathrm{B}}$ of $(\mathrm{a}) 0.2 \%$, (b) $0.5 \%$ and (c) $2.0 \%$. 


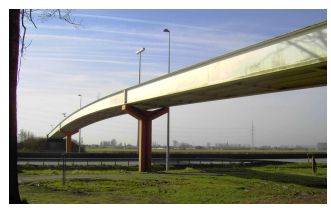

(b)

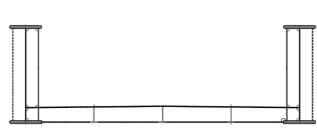

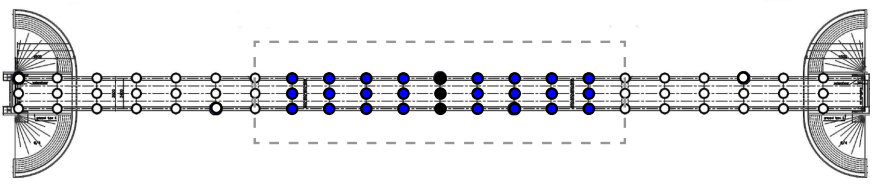

(c)

Figure 5: (a) Eeklo footbridge, (b) cross section and (c) top view with walking area (box) and stationary person locations (solid). 
(a)

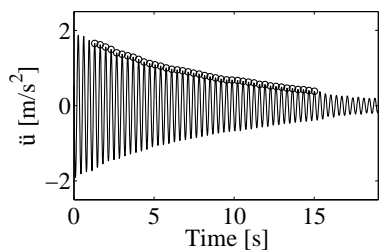

(d)

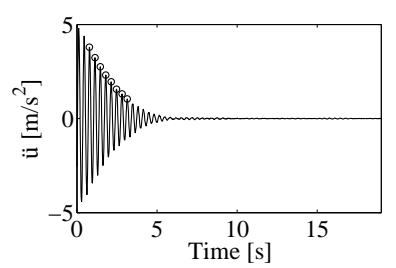

(b)

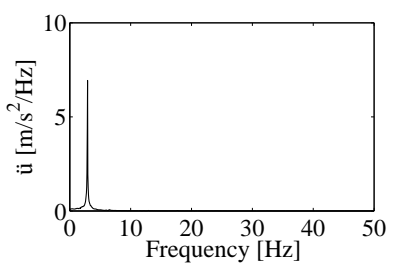

(e)

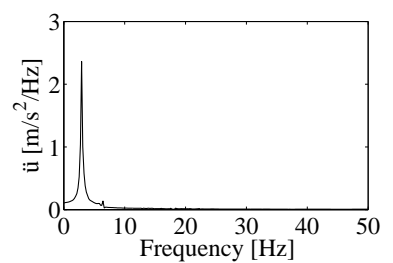

(c)

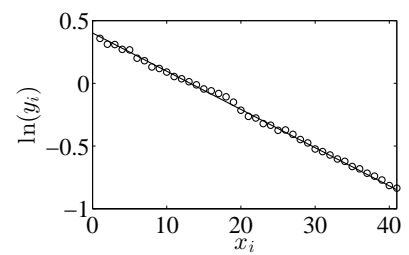

(f)

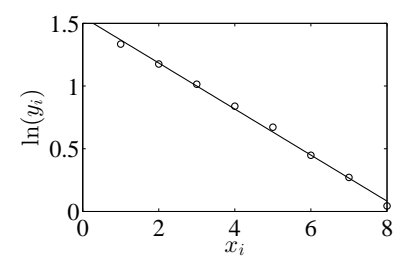

Figure 6: Measured vertical free vibration response of the footbridge at midspan, dominated by the contribution of the vertical bending mode $\left(f_{2}\right)$, with 15 human persons adopting the posture with $(\mathrm{a}-\mathrm{c})$ straight legs and $(\mathrm{d}-\mathrm{f})$ slightly bent legs: (a,d) time series with selected peak values, (b,e) corresponding amplitude spectrum and (c,f) natural logarithm and best-fit line of peak amplitudes $\left(y_{i}\right)$ and number of cycles $\left(x_{i}\right)$. 

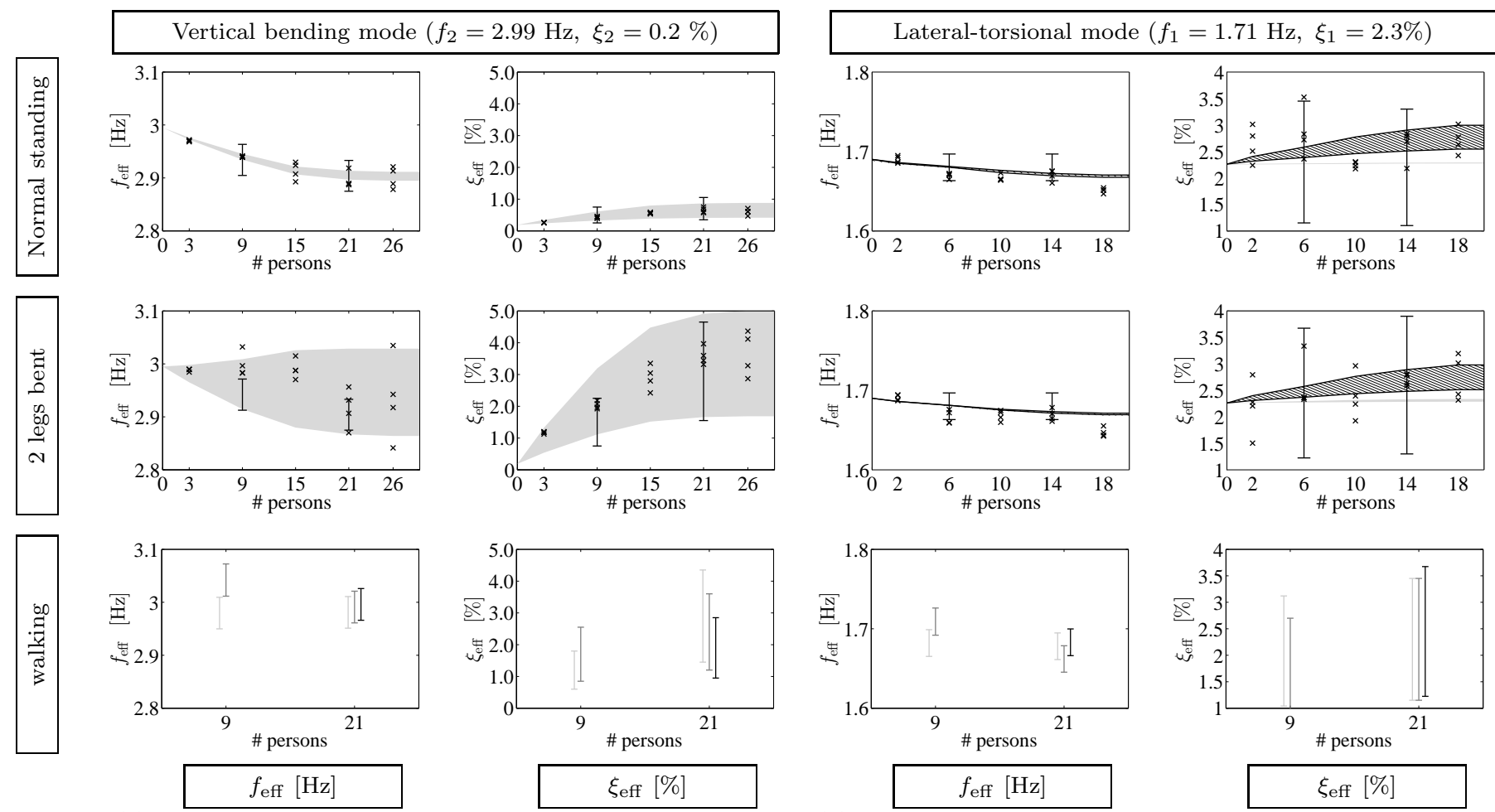

(a)
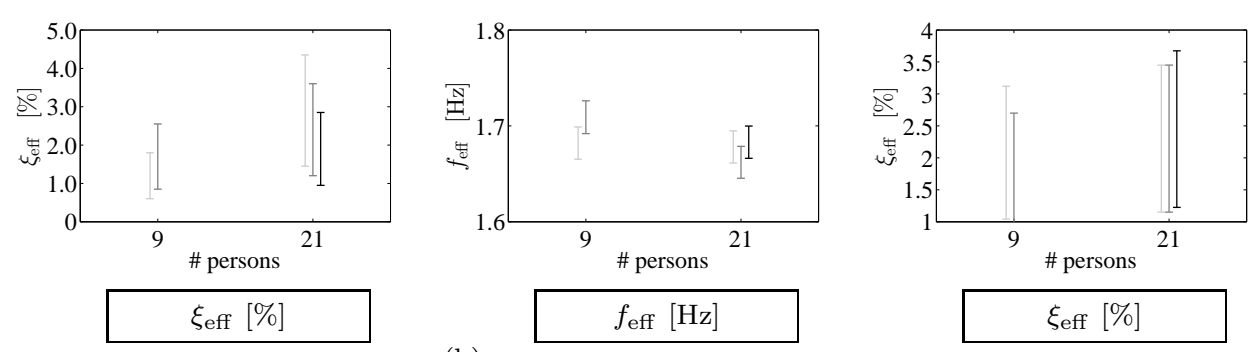

Figure 7: The effective natural frequency $f_{\text {eff }}$ and damping ratio $\xi_{\text {eff }}$ of the vertical bending mode (a) and the fundamental lateraltorsional mode (b): the $90 \%$ confidence region computed by the coupled crowd-structure model (grey area), including in addition horizontal interaction (hatched), identified from the free decay analysis $(\times)$ and identified from the OMA (confidence interval), for a normal standing posture, 2 legs bent and for persons walking at a low (light grey), normal (dark grey) and fast (black) speed. 

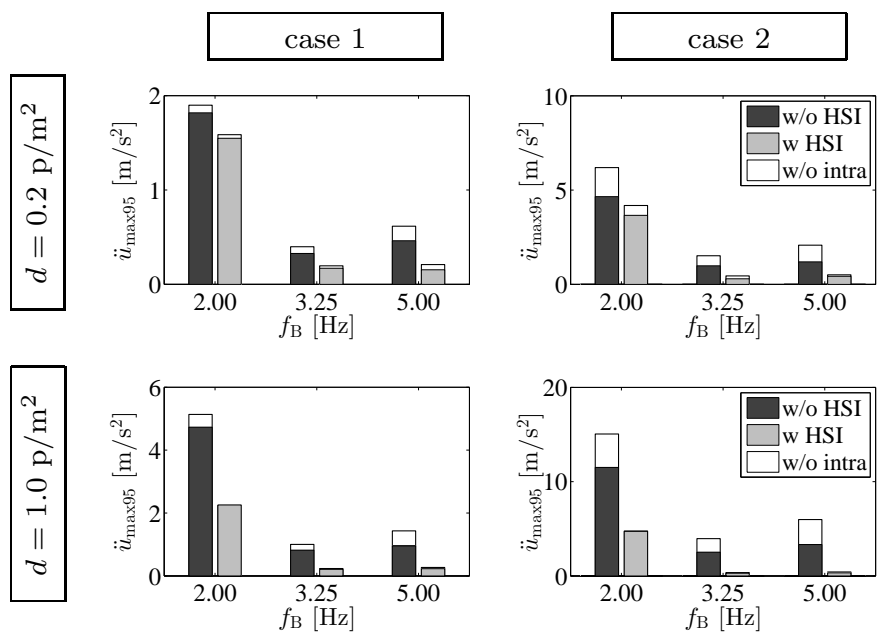

Figure 8: The 95 percentile value of the maximum acceleration at midspan $\left(\ddot{u}_{\max 95}\right)$ with the step frequencies of the pedestrians set to follow a Gaussian distribution (case 1) or all identical (case 2), disregarding HSI (dark grey), taking into account HSI (light grey) and disregarding intra-person variability (white). 

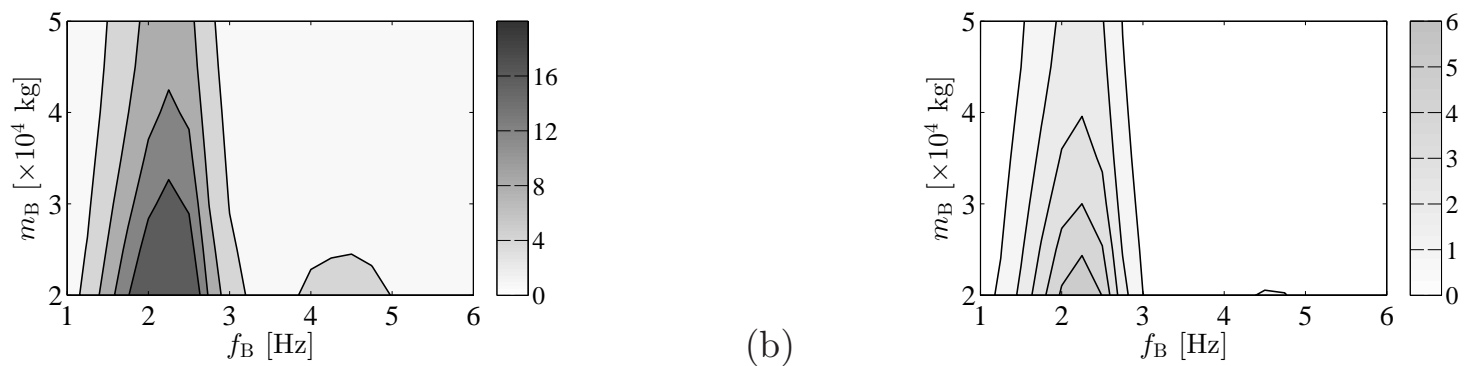

Figure 9: The 95 percentile value of the maximum acceleration predicted by the MF-model $\left(\ddot{u}_{\mathrm{F}, \max 95}\left[\mathrm{~m} / \mathrm{s}^{2}\right]\right)$ in terms of the natural frequency $\left(f_{\mathrm{B}}\right)$ and modal mass $\left(m_{\mathrm{B}}\right)$ of the footbridge with $\xi_{\mathrm{B}}=0.5 \%$ for: (a) a low $\left(0.2\right.$ persons $\left./ \mathrm{m}^{2}\right)$ and (b) a high (1.0 persons $\left./ \mathrm{m}^{2}\right)$ pedestrian density. 

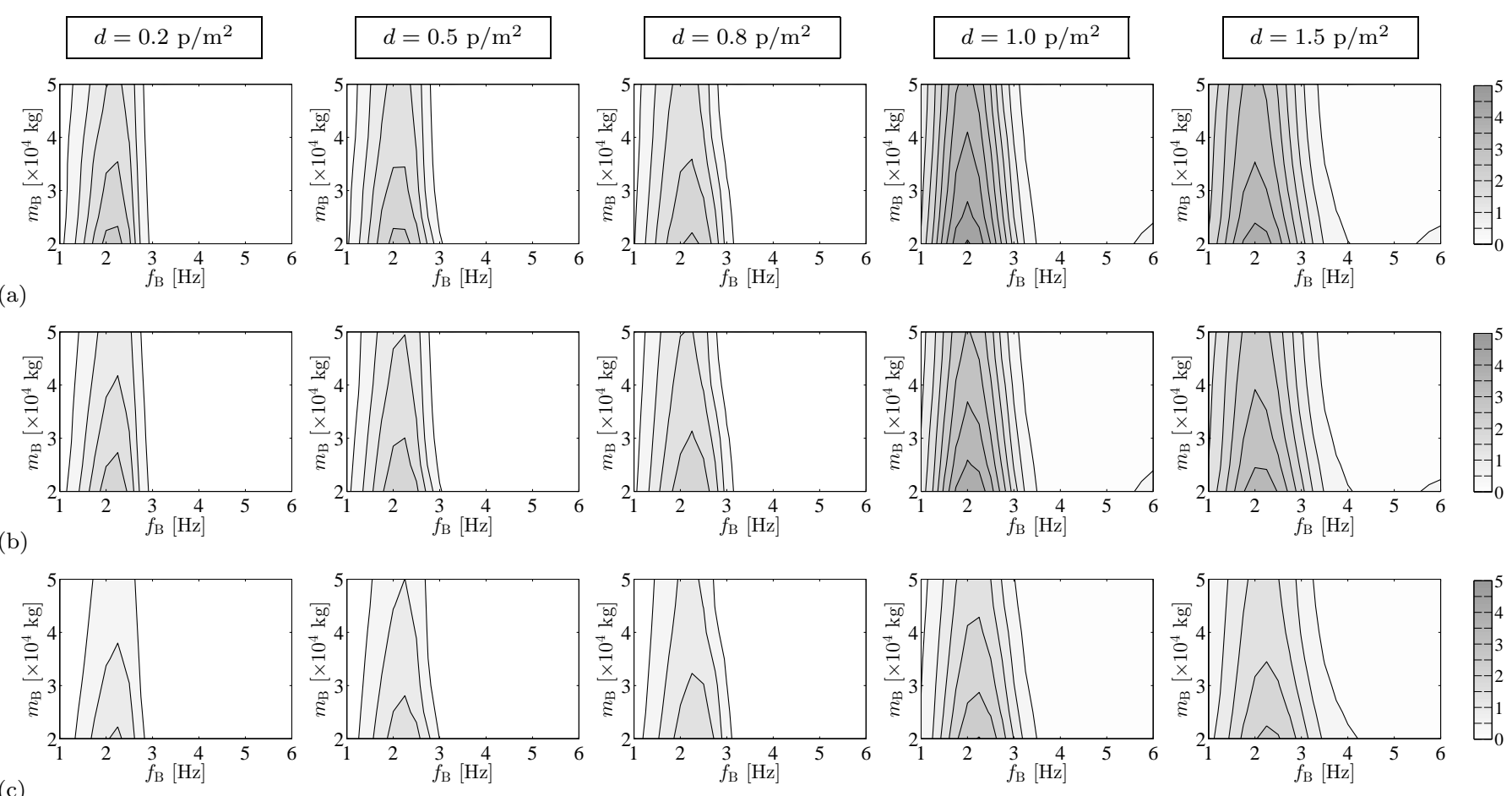

Figure 10: The 95 percentile values of the maximum acceleration levels at midspan of the bridge $\left(\ddot{u}_{\mathrm{HSI}}, \mathrm{max} 95\left[\mathrm{~m} / \mathrm{s}^{2}\right]\right)$ predicted by the PMC-model for low $\left(d<1\right.$ persons $\left./ \mathrm{m}^{2}\right)$ and high $\left(d \geq 1\right.$ persons $\left./ \mathrm{m}^{2}\right)$ pedestrian densities, for a structural inherent damping ratio of (a) $\xi_{\mathrm{B}}=0.2 \%$, (b) $\xi_{\mathrm{B}}=0.5 \%$ and (c) $\xi_{\mathrm{B}}=2.0 \%$, in terms of the natural frequency $\left(f_{\mathrm{B}}\right)$ and modal mass $\left(m_{\mathrm{B}}\right)$ of the footbridge. 

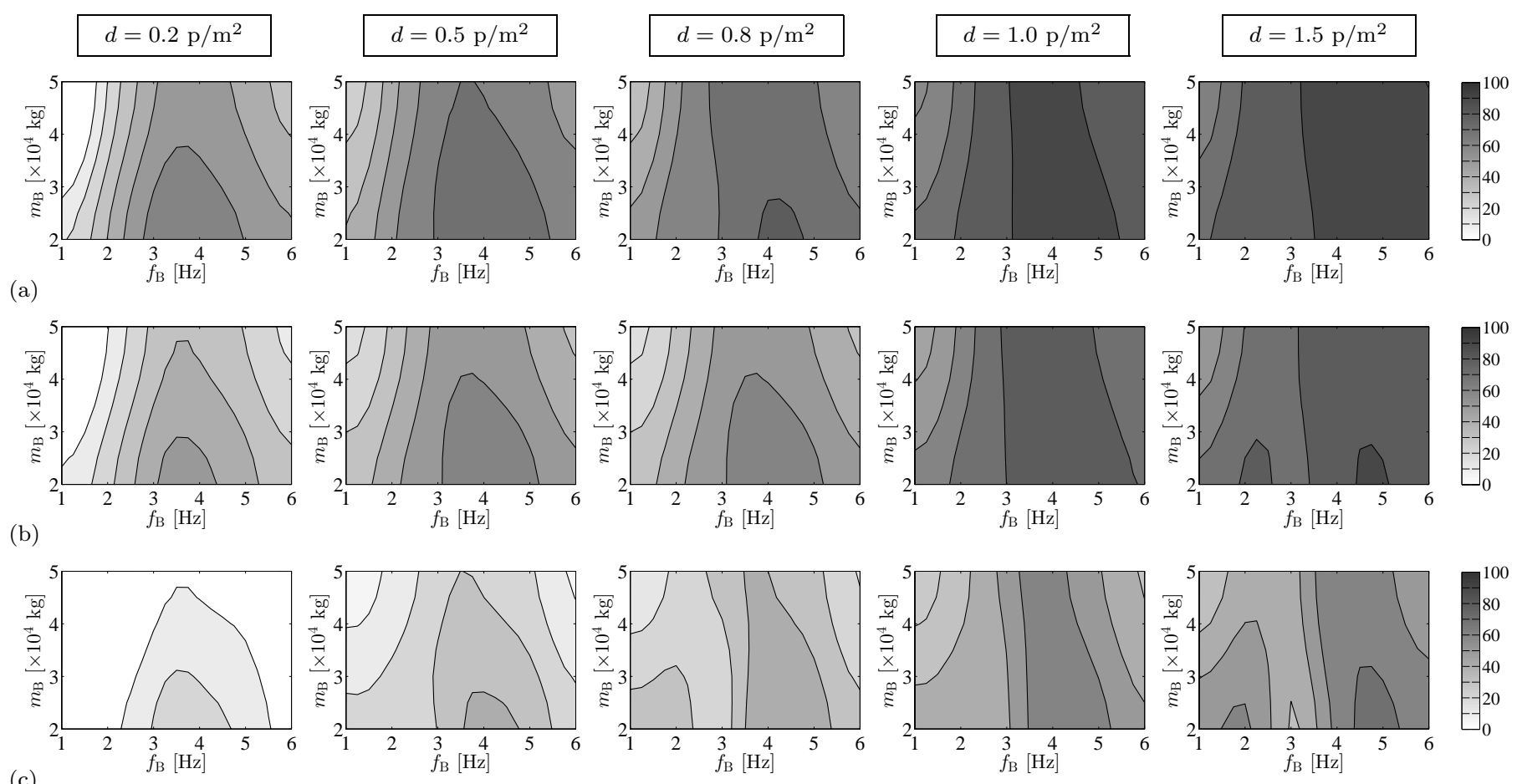

Figure 11: The reduction factor $R_{\mathrm{HSI}}[-]$ derived for low $\left(d<1\right.$ persons $\left./ \mathrm{m}^{2}\right)$ and high $\left(d \geq 1\right.$ persons $\left./ \mathrm{m}^{2}\right)$ pedestrian densities, for a structural inherent damping ratio of (a) $\xi_{\mathrm{B}}=0.2 \%$, (b) $\xi_{\mathrm{B}}=0.5 \%$ and (c) $\xi_{\mathrm{B}}=2.0 \%$, in terms of the natural frequency $\left(f_{\mathrm{B}}\right)$ and modal mass $\left(m_{\mathrm{B}}\right)$ of the footbridge. 


\begin{tabular}{llll}
\hline Name & Abbreviation & $\begin{array}{l}\text { fixed-driver } \\
\text { term }\end{array}$ & Human-structure interaction \\
\hline Stationary force model & SF & stationary & disregarded \\
Moving force model & MF & moving & disregarded \\
Moving force model with added mass & MFAM & moving & uniformly distributed crowd mass \\
Pseudo moving crowd model & PMC & moving & stationary human body models \\
Moving crowd model & MC & moving & moving human body models \\
\hline
\end{tabular}

Table 1: The main characteristics of the numerical models considered to predict the response of the footbridge to crossing pedestrians. 


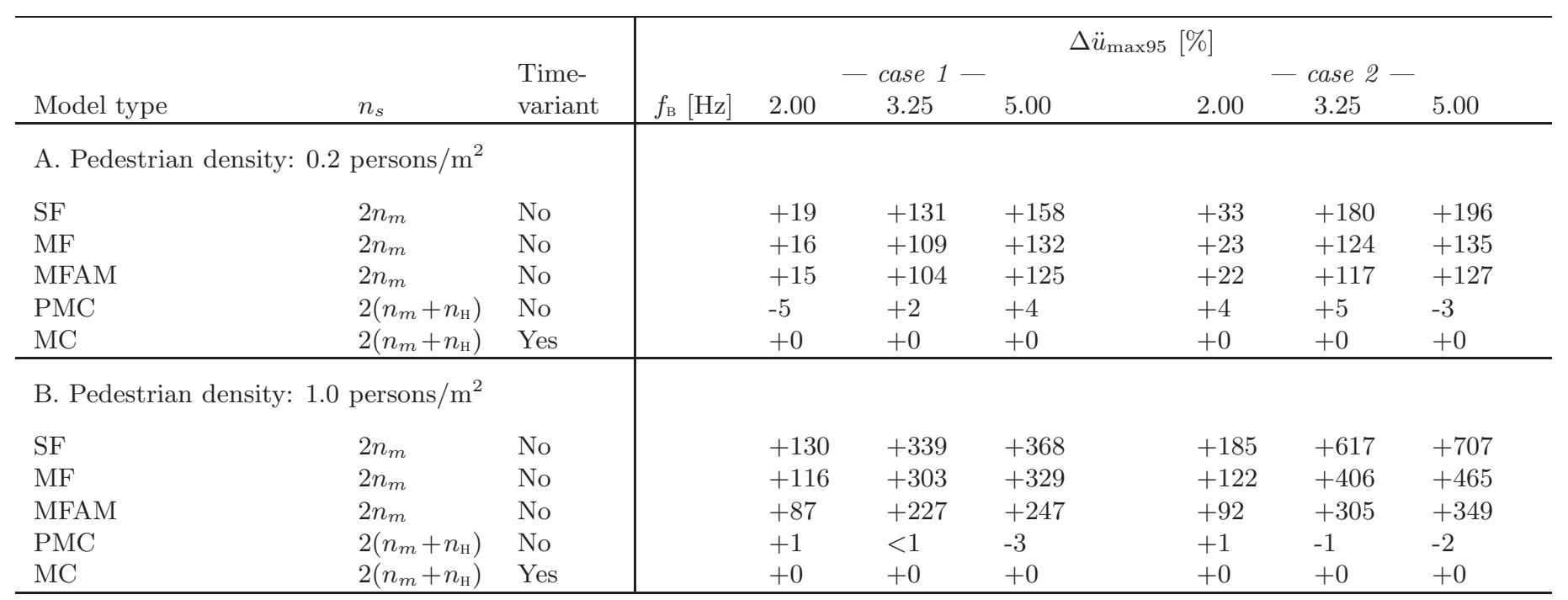

Table 2: The system order $n_{s}$, type and the predicted 95 percentile value of the maximum $\left(\ddot{u}_{\text {max } 95}\right)$ acceleration, relative to the one of the moving crowd model (MC). 